Portland State University

PDXScholar

Mechanical and Materials Engineering Faculty

Publications and Presentations

$11-2006$

\title{
Capillary-Driven Flows Along Rounded Interior Corners
}

Yongkang Chen

Portland State University

Mark M. Weislogel

Portland State University, weisloge@pdx.edu

Cory L. Nardin

Portland State University

Follow this and additional works at: https://pdxscholar.library.pdx.edu/mengin_fac

Part of the Aerodynamics and Fluid Mechanics Commons, Fluid Dynamics Commons, and the Materials Science and Engineering Commons

Let us know how access to this document benefits you.

\section{Citation Details}

YONGKANG CHEN, MARK M. WEISLOGEL and CORY L. NARDIN (2006). Capillary-driven flows along rounded interior corners. Journal of Fluid Mechanics, 566, pp 235-271 doi:10.1017/S0022112006001996

This Article is brought to you for free and open access. It has been accepted for inclusion in Mechanical and Materials Engineering Faculty Publications and Presentations by an authorized administrator of PDXScholar. Please contact us if we can make this document more accessible: pdxscholar@pdx.edu. 


\title{
Capillary-driven flows along rounded interior corners
}

\author{
By YONGKANG CHEN, MARK M. WEISLOGEL \\ AND CORY L. NARDIN \\ Department of Mechanical and Materials Engineering, Portland State University, \\ P.O. Box 751, Portland, OR 97207, USA
}

(Received 30 October 2005 and in revised form 3 April 2006)

The problem of low-gravity isothermal capillary flow along interior corners that are rounded is revisited analytically in this work. By careful selection of geometric length scales and through the introduction of a new geometric scaling parameter $\bar{T}_{c}$, the Navier-Stokes equation is reduced to a convenient $\sim O(1)$ form for both analytic and numeric solutions for all values of corner half-angle $\alpha$ and corner roundedness ratio $\lambda$ for perfectly wetting fluids. The scaling and analysis of the problem captures much of the intricate geometric dependence of the viscous resistance and significantly reduces the reliance on numerical data compared with several previous solution methods and the numerous subsequent studies that cite them. In general, three asymptotic regimes may be identified from the large second-order nonlinear evolution equation: (I) the 'sharp-corner' regime, (II) the narrow-corner 'rectangular section' regime, and (III) the 'thin film' regime. Flows are observed to undergo transition between regimes, or they may exist essentially in a single regime depending on the system. Perhaps surprisingly, for the case of imbibition in tubes or pores with rounded interior corners similarity solutions are possible to the full equation, which is readily solved numerically. Approximate analytical solutions are also possible under the constraints of the three regimes, which are clearly identified. The general analysis enables analytic solutions to many rounded-corner flows, and example solutions for steady flows, perturbed infinite columns, and imbibing flows over initially dry and prewetted surfaces are provided.

\section{Introduction and overview}

Capillary flows are defined here as spontaneous interfacial flows driven by surface tension, container geometry, and surface wettability, for which the impact of gravity is negligible. Such flows are fundamental to a myriad transport processes in both nature and industry and range from microscale flows $\lesssim O(1 \mathrm{~mm})$ in porous media on Earth to macroscale flows $\lesssim O(1 \mathrm{~m})$ in large liquid fuel tanks aboard spacecraft. The 'interior corner' geometry is the focus of this research as it is commonly employed in systems where the corner serves as a conduit to guide passively a particular fluid phase in a desired manner. In many instances, the interior corners are not perfectly sharp but rather possess a degree of roundedness due to formation or fabrication, as shown in figure 1. While flows in perfectly sharp interior corners have been studied extensively, the impact of corner imperfections such as corner roundedness has not been fully characterized analytically. And, for example, because significant roundedness can prevent certain imbibition within porous materials and even slight roundedness can prevent the spread of liquid drops in otherwise wettable corners, it 


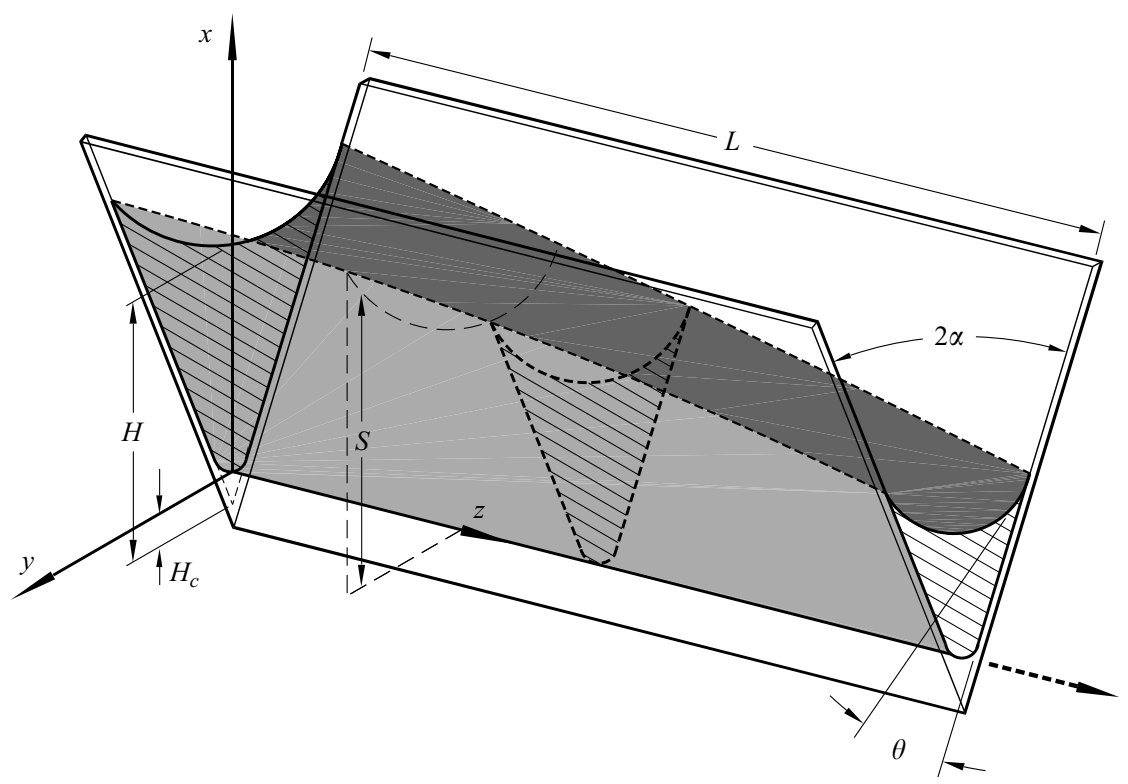

FIGURE 1. A fluid column in a rounded corner of angle $2 \alpha$. The coordinate system is aligned such that the $z$-axis is along the vertex of the interior corner. The three-dimensional surface profile is $S(y, z, t)$. The contact angle is $\theta$.

may be critical to system design and analysis to assess quantitatively the impact of the degree of roundedness on the process.

In general, and as depicted in figure 1, capillary flows along interior corners tend to be slender. This is certainly the case for flows along high-aspect-ratio conduits such as polygonal-section capillaries (Weislogel 2001a) and microchannels (Cubard \& Ho 2004; Tchikanda, Nilson \& Griffiths 2004), flows along surface grooves (Mann et al. 1995), and flows within highly angular porous or baffled structures at low saturation (Al-Futaisi \& Patzek 2002; Weislogel \& Collicott 2004). The slenderness of the liquid column allows the application of lubrication theory, where the problem simplifies to one of first solving for the average $z$-component velocity $\langle w\rangle$ at any cross-section in the $(x, y)$-plane and then using this result and a mass balance to derive an evolution equation for flow along the $z$-axis. A solution for the interface shape as a function of boundary conditions and time is generally obtained, from which the most important features of the flow can be determined. Solutions of this type form the basis from which problems of significantly greater complexity may be addressed.

The first problem is referred to here as the two-dimensional 'crossflow problem'. The domain is depicted in figure 2 for a capillary-dominated surface in a perfectly sharp interior corner. This portion of the solution requires two steps: (i) the velocity distribution $w(x, y)$ must be determined and then (ii) it must be integrated over the domain to compute the average velocity $\langle w\rangle(z, t)$ for the section. The crossflow problem can be solved analytically for select values of corner half-angle $\alpha$ and wetting angle $\theta$ (i.e. $\alpha=\theta=\pi / 4$ ), asymptotically for wide- and narrow-corner halfangle $(\alpha \rightarrow \pi / 2$ and $\alpha \ll 1$, respectively), or numerically for all values of $\alpha$ and $\theta \cdot \dagger$

$\dagger$ Conformal mappings using Schwarzian S-functions (Nehari 1975) permit the exact analytic expression of solutions to the crossflow problem for $w(x, y)$. However, numerical transformations are presently necessary to determine velocity profiles prior to the integration of $w(x, y)$ over the domain to compute $\langle w\rangle$. 


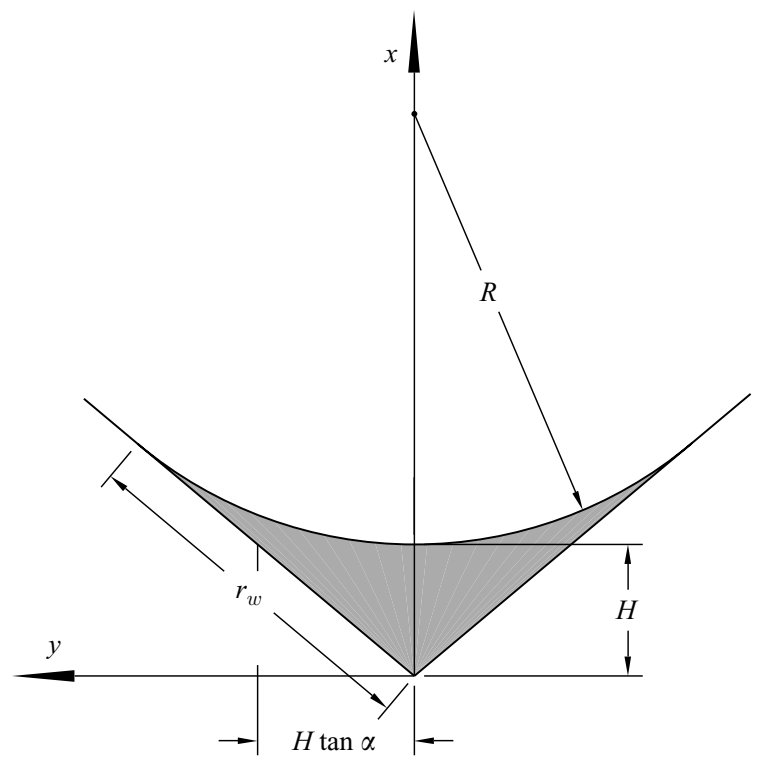

FIGURE 2. Length scales adopted by different studies: $r_{w}$ is used for all lengths by Ayyaswamy, Catton \& Edwards (1974), $R$ for all lengths by Ransohoff \& Radke (1988), $x \sim H$ and $y \sim H \tan \alpha$ by Weislogel \& Lichter (1998).

For all solution methods, the integration of the velocity field $w(x, y)$ determines a dimensionless geometric function that primarily characterizes the viscous resistance of the flow and is often referred to as a flow-resistance function, friction factor, or hydraulic conductance. Other names are used as well, or none at all. For the sharp-corner geometry, the flow-resistance function depends on $\alpha$ and $\theta$ only. The introduction of corner roundedness introduces a dependence of the flow resistance on fluid depth $h$ in addition to corner roundedness, $\alpha$, and $\theta$.

As will be demonstrated herein, a well-considered scaling of the crossflow problem can lead to a flow-resistance function that is narrowly confined for all the geometric parameters of the problem. This feature significantly weakens the dependence on numerical data and greatly expands the prospects of analysis. Previous numerical solutions may be re-interpreted in this light and a brief review of the corner-flow literature is therefore discussed here. For example, in an often overlooked investigation, Ayyaswamy et al. (1974) solved the crossflow problem numerically for the sharp-corner problem and obtained a dimensionless friction factor $K$ using the Galerkin method in radial coordinates. All lengths in their domain are scaled by the wetted wall length $r_{w}$ identified in figure 2 . Their friction factor $30 \lesssim K(\alpha, \theta) \lesssim 57$ is bounded for all values of $\alpha$ and $\theta$ but not as tightly as might be achieved using a more comprehensive scaling (Weislogel \& Lichter 1998). As part of a later numerical study, Ransohoff \& Radke (1988) solved the same numerical problem as Ayyaswamy et al. (1974). They used a radial coordinate system for their solution domain with the origin at the centre of the free-surface curvature. The radial coordinate is non-dimensionalized by the radius of curvature of the free surface, $R$, which is also shown in figure 2 . The resulting flow-resistance function $6 \leqslant \beta(\alpha, \theta) \leqslant \infty$ is unbounded (Weislogel \& Lichter 1998) for all values of $\alpha$ and $\theta$. Subsequent analyses employing $\beta$ (Dong \& Chatzis 1995; Tuller \& Or 2001) were forced to curve-fit $\beta$ in the domain of interest, which often spans one or two orders of magnitude if not more. Significantly, $\beta$ is 
most commonly employed in porous-media studies despite its unbounded nature. $\dagger$ As a result, such investigations are in turn heavily dependent on tabulated numerical data.

Romero \& Yost (1996) obtained an accurate analytical approximation for their unnamed resistance coefficient $0 \leqslant \Gamma \leqslant \infty$ that enabled a well-constructed similarity solution for certain capillary flows along interior corners. Weislogel \& Lichter (1998) chose length scales $x \sim H$ and $y \sim H \tan \alpha$ (see figure 2) to compute a velocity scale $W$ used to non-dimensionalize the crossflow problem. An asymptotic analysis was then pursued providing the limiting values for functions such as $K, \beta$, and $\Gamma$ by introducing a preferred geometric-flow-resistance function $1 / 8 \lesssim F_{i}(\alpha, \theta) \leqslant 1 / 6$, which is narrowly bounded for all values of $\alpha$ and $\theta$. The fact that $F_{i}$ is a narrowly confined function implies that the simple scaling accounts for much of the geometric dependence of the crossflow problem and that for many practical problems $F_{i}$ may be treated as an $O(1)$ constant, say, $\simeq 1 / 7$. Closed-form analytical solutions employing $F_{i}$ are concise, where geometric dependencies may be distinguished clearly and often by inspection and where efficient geometric optimization schemes are straightforward. The $F_{i}$ formulation of the crossflow problem also yields perhaps the most compact forms for pore-level transport in large porous media models (Tuller \& Or 2001). The published resistance coefficients may be expressed in terms of $F_{i}$ : thus $K=8\left(F_{A} / f \sin \delta\right)^{2} / F_{i}$, $\beta=f^{2} / F_{i} \sin ^{2} \alpha$, and $\Gamma=F_{A} F_{i} \sin ^{2} \alpha(\tan \alpha / f \sin \delta)^{4}$, where $f, \delta$, and $F_{A}$ are compact analytic geometric functions defined herein.

Ransohoff \& Radke (1988) also investigated rounded corners where the rounded portion of the corner is concentric with the free surface (Ransohoff \& Radke 1988, figure 4). Their flow-resistance function $\beta$ was determined numerically for a selection of corner half-angles, contact angles, and degrees of roundedness - the last of which was measured using a ratio that employs the depth of the fluid $h$. The corner roundedness by this definition is actually a local quantity, different from that defined in this work, and varies along the flow direction if the depth of the fluid changes. Thus $\beta=\beta\left(\alpha, \theta, R_{c}, h\right)$ for a given flow, where $R_{c}$ and $h$ are indicated for the roundedcorner crossflow problem in figure 3. Employing the rounded-corner local scaling, to be introduced herein, it can be shown that $3.2 \lesssim \beta \leqslant \infty$ for all values of $\alpha, \theta, R_{c}$, and $h$. Analysis employing $\beta$ for rounded-corner flows must convert the tabulated results to forms that can be used for the global problem. This approach was adopted by Zhou, Blunt \& Orr (1997) to study hydrocarbon drainage over a layer of water forming a virtual rounded corner with the solid walls, which form sharp interior corners. It is observed that the flow along rounded corners is complicated by the fact that the flow resistance varies dramatically in the flow direction, as identified by Dong \& Chatzis (1995).

In solving for the imbibition of liquid in square capillary tubes with rounded interior corners, Dong \& Chatzis (1995) used the approach of Ransohoff and Radke to calculate the flow resistance with a more practical definition of corner roundedness. Furthermore, they obtained analytic flow solutions on the basis of results given by Lenormand \& Zarcone (1984), who used the hydraulic-diameter approach to solve the flow in sharp corners. The hydraulic-diameter approach does provide an all-analytic solution to the crossflow problem but it also introduces errors up to more than $100 \%$, as reported by Ransohoff \& Radke (1988) and confirmed by subsequent comparative

$\dagger$ As of this writing, a Web of Science search of the Science Citation Index identified 67 papers citing Ransohoff \& Radke (1988) and using $\beta$ and only two citing Ayyaswamy et al. (1974) and using $K$. 


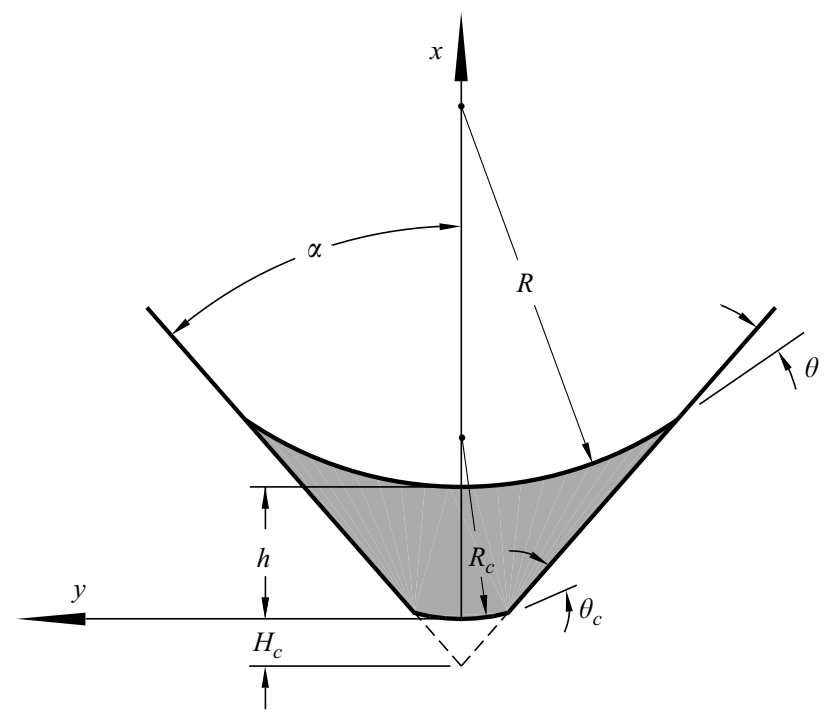

FiguRE 3. Dimensional crossflow problem. $R_{c}$ is the radius of the rounding arc of the corner meeting the planar walls at incident angle $\theta_{c}$. $R$ is the radius of curvature of the free surface and $\theta$ is the contact angle. In Ransohoff $\&$ Radke (1988), the rounding arcs of the corner and of the free surface are concentric, i.e. $R_{c}=R+h$, so that $\theta_{c}=\pi / 2-\arcsin (R \cos \theta /(R+h))$, whereas in this study $\theta=\theta_{c}$.

flow-rate calculations. A flow-resistance function $F_{\text {hyd }}$ based on the hydraulic diameter (Weislogel 1996) varies widely, $1 / 288 \leqslant F_{\text {hyd }} \leqslant 1 / 8$, for all values of $\alpha$ and $\theta$ and so this is not a preferred analytic approach in the light of alternatives.

Further reviews of interior-corner capillary flows may be cited (Weislogel 2003; Darhuber \& Troian 2005). Specific problems addressed in the literature include capillary imbibition and drainage for oil recovery (Zhou et al. 1997), flows in porous media (Lenormand \& Zarcone 1984), transport in groundwater systems (Tuller \& Or 2001), drainage flows in angular capillaries (Bico \& Quéré 2002), capillary flows in microfluidic devices (Peterson \& Ha 1998; Kim \& Whitesides 1997), and large-lengthscale capillary flows in vaned containers in microgravity environments (Weislogel \& Collicott 2004). The governing equations and solutions are also closely related to those of foam-drainage problems, and a large literature exists on this subject (Verbist et al. 1996; Cox \& Verbist 2003).

\section{Scope}

While previous studies provide useful solutions for capillary flows along sharp corners, there remains an unnecessarily strong dependence on the crossflow numerical data apparently because researchers are unaware of more analytical forms for the flow resistance. This shortcoming is most critical for rounded-corner flows, where an analytic form for the flow resistance as a function of corner roundedness and fluid depth is missing. The goal of the present work is to provide this quantity by extending the scaling and solution approach of Weislogel \& Lichter (1998) (referred to herein as $\mathrm{W} \& \mathrm{~L}$ ) to the rounded-corner problem and so to obtain closed-form analytical expressions from which such systems might be more efficiently designed and analyzed. 
In this paper, early and ample attention is paid to scaling and non-dimensionalization in a manner that leads to a more complete analytic description of the problem than that of previous investigations. Asymptotic analytic solutions are then provided for a new, 'narrowly confined', $\sim O(1)$, local geometric flow-resistance function $\langle\bar{w}\rangle^{*}$ for rounded corners in several geometric limits. (This function $\langle\bar{w}\rangle^{*}$ is not defined until (3.20) and is probably better described as a local normalized corner-axis-dependent average flow velocity.) The asymptotic results provide a benchmark for numerical solutions which are used to compute $\langle\bar{w}\rangle^{*}$ for a wide range of geometric parameters. The numerical results confirm the scaling arguments. A global evolution equation is then derived for the rounded-corner flow, which may be reduced to three asymptotic capillary corner-flow regimes. Steady solutions and infinite-fluid-column solutions for such flows are then presented. A similarity equation for capillary rise (imbibition) in containers with rounded interior corners is derived and solved, and key closed-form solutions are given. Further solutions considering finite advancing-front curvature are discussed and solved.

Only fluids that satisfy the Concus-Finn corner-wetting condition are addressed in this work. For sharp corners (Concus \& Finn 1969) the Concus-Finn condition requires that $\theta<\pi / 2-\alpha$; this condition produces an under-pressure in the fluid that draws it spontaneously into and along the corner. A rounded corner may prevent such a flow, depending on the system, i.e. the degree of roundedness, system geometry, contact angle, liquid volume, etc. Concus \& Finn (1990) provided examples of critical corner wetting in infinite cylinders with rounded rectangular sections. In situations where the contact angle $\theta$ is fixed, a corresponding critical (maximum) roundedness for every corner can be identified above which no spontaneous corner flow can take place. For containers or channels with several corners, the critical roundedness of a given corner is affected by the roundedness of the others. Thus, for complex geometries, a concerted effort may be required just to determine whether the rounded corner is actually wetted. The impact of contact-angle hysteresis can complicate this picture dramatically (Kistler 1993; Concus, Finn \& Weislogel 1996).

For the special case of a spreading drop in a sharp corner (Weislogel \& Lichter 1996) the fluid spreads to $z= \pm \infty$ provided that $\theta<\pi / 2-\alpha$. For $\theta>0$, any degree of corner roundedness will prevent such a spread and will confine the axial domain of the drop to $|z| \lesssim V(\cos \theta-\sin \alpha)^{2} /\left[(2 \theta-\sin 2 \theta) H_{c}^{2} \sin ^{2} \alpha\right]$, where $V$ is the drop volume and $H_{c}$ is the height of the rounded corner as shown in figure 1. Only in the case $\theta=0$ will a liquid drop spread indefinitely along a rounded interior corner. The behaviour of such perfectly wetting fluids is the primary focus of this work. Partial-wetting systems will be reported in a separate work.

\section{Analysis}

For the system shown in figure 1 , the characteristic geometric quantities of the problem are the lengths $H, H_{c}$, and $L$ and the angles $\alpha$ and $\theta$. The relevant parameters are collected in table 1 . Note that $H$ is the characteristic height of the meniscus in the $(x, y)$-plane measured from the virtual vertex of the corner as if it were sharp. The analysis that follows requires primarily the constraint of a slender column, $\epsilon_{c}^{2} \ll 1$, but also the restriction of low inertia, $\mathscr{R} \ll 1$, and a dominant cross-stream curvature, $\epsilon_{c}^{2} f \ll 1$. Here

$$
\epsilon_{c} \equiv(1-\lambda)(H / L)
$$

with

$$
\lambda \equiv H_{c} / H
$$


$\sigma$, surface tension $\left(\mathrm{N} \mathrm{m}^{-1}\right)$

$\rho$, density $\left(\mathrm{kg} \mathrm{m}^{-3}\right)$

$\mu$, dynamic viscosity $\left(\mathrm{kg} \mathrm{m}^{-1} \mathrm{~s}^{-1}\right)$

$\theta$, contact angle (rad)

$\alpha$, corner half-angle (rad)

$\delta, \pi / 2-\alpha-\theta$, see figure 4 (rad)

$f$, cross-flow interface curvature function (3.5)

$f_{c}$, corner curvature function (3.10)

$F_{A}$, cross-flow area function (3.22)
$L$, fluid-column length scale (m)

$H$, meniscus height scale $(\mathrm{m})$

$H_{c}$, rounded corner height $(\mathrm{m})$

$h$, meniscus height $(\mathrm{m})$

$\epsilon_{c}$, fluid-column slenderness ratio (3.1)

$\lambda$, corner roundedness (3.2)

$\langle\bar{w}\rangle^{*}$, dimensionless local average velocity

$\langle w\rangle^{*}$, dimensionless global average velocity

TABLE 1. Selection of relevant physical and geometrical parameters.

and

$$
\mathscr{R} \equiv \epsilon_{c}^{2}(1-\lambda)^{3} \frac{\sigma \rho H}{f \mu^{2}}\left(\frac{T_{c}^{2}}{1+T_{c}^{2}}\right)^{2},
$$

where $\sigma, \rho$, and $\mu$ are respectively the fluid surface tension, density, and viscosity and

$$
R=\left(h+H_{c}\right) f,
$$

with

$$
f=\frac{\sin \alpha}{\cos \theta-\sin \alpha} .
$$

The parameter $\mathscr{R}$ is a measure of inertia and serves as a strongly geometrydependent capillary-flow Reynolds number for the rounded-corner problem (akin to the Suratman number $S u=\sigma \rho H / \mu^{2}$ ). This parameter is comparable with that defined for the sharp-corner problem by W\&L. $T_{c}$ is a ratio of the $y$ - and $x$-coordinate length scales to be defined. Under these restrictions it can be shown that the Navier-Stokes equation reduces to the zeroth-order $z$-component equation

$$
\frac{1}{\mu} \frac{\partial P}{\partial z}=\frac{\partial^{2} w}{\partial x^{2}}+\frac{\partial^{2} w}{\partial y^{2}}
$$

where $P$ is the under-pressure in the fluid,

$$
P=-\frac{\sigma}{R}=-\frac{\sigma}{\left(h+H_{c}\right) f},
$$

due to the surface curvature (as addressed by W\&L; see also Weislogel 1996) and again $w$ is the corner-axis $z$-component velocity. Equation (3.6) is the dimensional 'crossflow problem' discussed above and is subject to the boundary conditions of no slip along the walls, no shear stress along the free surface, and the contact angle condition at the contact line (see figure 3 ). To determine the flow along the $z$-coordinate, $w(x, y)$ must be determined and integrated over the local crossflow area $A$ to determine the average velocity $\langle w\rangle$, which is then substituted into a mass-balance equation expressed dimensionally as

$$
\frac{\partial A}{\partial t}=-\frac{\partial}{\partial z}(A\langle w\rangle),
$$

from which an evolution equation for $h(z, t ; \alpha, \theta, \lambda)$ may be derived (Lenormand \& Zarcone 1984; Ransohoff, Gauglitz \& Radke 1987). The geometry of the crossflow section for the rounded-corner problem can change dramatically depending on $H$, $H_{c}, \alpha$, and $\theta$. 


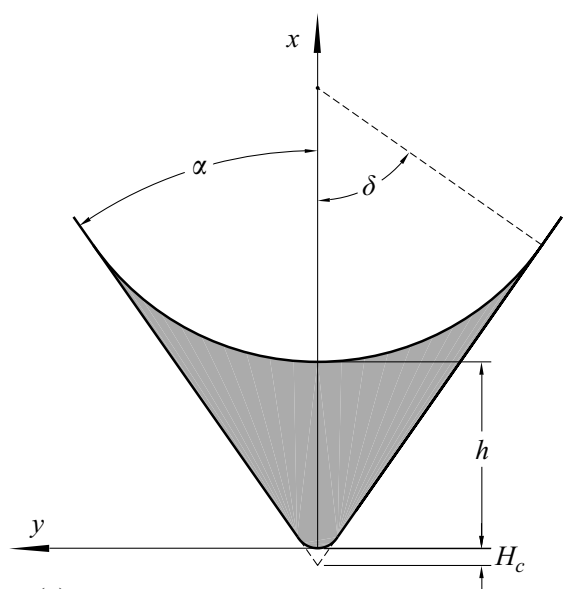

(a)

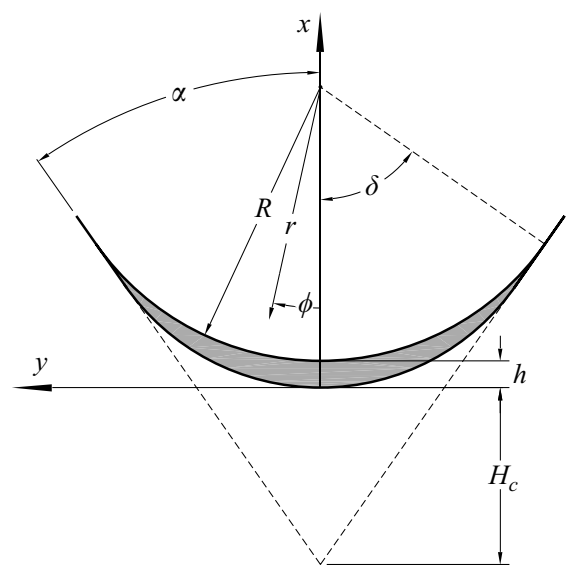

(b)

FIGURE 4. Characteristic crossflow sections for fixed $\alpha$ and $\theta:(a)$ sharp-corner-like section and $(b)$ crescent-like section.

\subsection{Scaling and non-dimensionalization}

\subsubsection{Local scaling}

Following the approach of W\&L, (3.6) is non-dimensionalized by a careful selection of scales for the rounded-corner problem. The coordinate system selected here places the intersection of the rounded corner and corner symmetry plane along the $z$-axis as pictured in figure 1 . The length of the liquid column along the $z$-direction is naturally scaled by $L$. Unfortunately, the introduction of corner roundedness adds some geometric complexity to the scaling of the $x$-and $y$-coordinates. These 'crossflow coordinates' require a local scaling. For example, possible characteristic cross-flow sections are shown in figures $4(a)$ and $4(b)$ for fixed $\alpha$ and $\theta$. Depending on the relative values of the characteristic fluid height $h \leqslant H-H_{c}$ and the virtual corner height $H_{c}$, the crossflow section can either appear sharp-corner-like as in figure 4(a), where $H_{c} /\left(h+H_{c}\right) \ll 1$, or crescent-like as in figure $4(b)$, where $H_{c} /\left(h+H_{c}\right) \rightarrow 1$. The geometry of the former is successfully scaled by local Cartesian lengths, i.e. $x \sim h$ and $y \sim\left(h+H_{c}\right) \tan \alpha$, as is the sharp-corner problem for $H_{c}=0$, where $x \sim H$ and $y \sim H \tan \alpha$ are found to be acceptable scales. However, the situation of figure 4(b) is more appropriately scaled using an $(r, \phi)$ cylindrical coordinate system with the origin at the centre of the free-surface curvature, i.e. $r \sim R+h, \Delta r \sim h$, and $\phi \sim \delta$, where $\delta \equiv \pi / 2-\alpha-\theta$. Employing both coordinate systems it is possible to use scale analysis to determine a single local Cartesian scaling that captures both limits, namely

$$
x \sim h \equiv \bar{x}_{s}
$$

and

$$
y \sim h \tan \alpha+H_{c} f_{c} \delta \equiv \bar{y}_{s},
$$

where $R_{c}=H_{c} f_{c}$ and again $R=\left(h+H_{c}\right) f, f_{c}$ characterizing the corner curvature and $f$ characterizing the fluid-interface curvature. These length scales are local scales determined by the local value of $h(z, t)$ and may be used to non-dimensionalize the crossflow (3.6). The notation $\bar{x}_{s}$, for example, in (3.9a) is used to denote an $h$ - and thus $z$-dependent $x$-coordinate length scale. Similarly, $\bar{y}_{s}$ is an $h$ - and thus $z$-dependent 


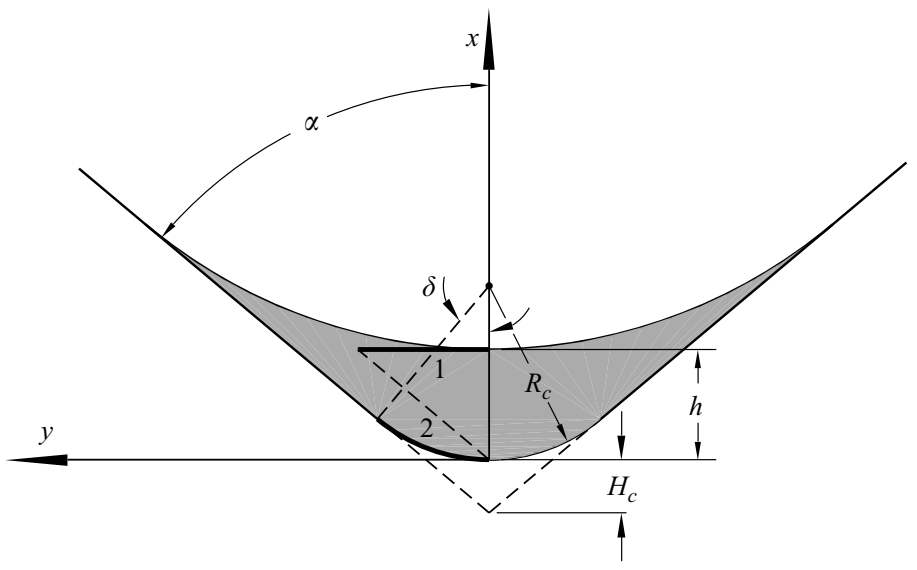

FiguRE 5. Length scales for the rounded-corner problem. The bold lines 1 and 2 are equal to $h \tan \alpha$ and $R_{c} \delta$, respectively.

\begin{tabular}{lll}
\multicolumn{1}{c}{ Lengths } & \multicolumn{1}{c}{ Velocities } & \multicolumn{1}{c}{ Other } \\
$\bar{x}^{*}=x / h$ & $\bar{u}^{*}=u / \epsilon_{c} \bar{W}$ & $\bar{A}^{*}=A / h^{2} \bar{T}_{c}$ \\
$\bar{y}^{*}=y / h \bar{T}_{c}$ & $\bar{v}^{*}=v / \epsilon_{c} \bar{W} \bar{T}_{c}$ & $\bar{t}^{*}=\bar{W} t / L$ \\
$\bar{z}^{*}=z^{*}=z / L$ & $\bar{w}^{*}=w / \bar{W}$ & $\overline{(\partial P / \partial z)^{*}}=1$ \\
$\bar{S}^{*}=S / h$ & $\langle\bar{w}\rangle^{*}=\langle w\rangle / \bar{W}$ & $\bar{T}_{c}=\tan \alpha+f \delta \bar{\lambda}$ \\
$\bar{h}^{*}=1$ & $\bar{W}=-\frac{h^{2}}{\mu} \frac{\partial P}{\partial z}\left(\frac{\bar{T}_{c}^{2}}{1+\bar{T}_{c}^{2}}\right)$ & $\bar{\lambda}=\lambda /\left[(1-\lambda) h^{*}\right]$
\end{tabular}

TABLE 2. Local non-dimensionalized dependent and independent variables.

$y$-coordinate length scale. The length scale $\bar{y}_{s}$ consists of the two lengths identified in figure 5, where it can be observed how the sharp-corner scaling is recovered for $H_{c} /\left(h+H_{c}\right) \rightarrow 0$ while the crescent-section scaling is recovered for $H_{c} /\left(h+H_{c}\right) \rightarrow 1$. As will be shown, these scales are appropriate for rounded corners that meet the planar walls of the corner at the same or nearly the same angle $\theta_{c}$ as the contact angle $\theta$. For identical angles $\theta=\theta_{c}$,

$$
f \equiv \frac{\sin \alpha}{\cos \theta-\sin \alpha}=f_{c} \equiv \frac{\sin \alpha}{\cos \theta_{c}-\sin \alpha} .
$$

The special case for rounded corners that are tangent to their planar walls, for which $\theta_{c}=0$, is the focus of this paper. Therefore the analyses presented are for perfectly wetted corners where $\theta=\theta_{c}=0$. For systems where $\theta \neq \theta_{c}$, or for other rounded-corner types, a more involved scaling approach may be taken.

\subsubsection{Local cross-flow equation}

The $z$-dependent length scales of (3.9a) and (3.9b) (see table 2) may be used to compute a $z$-dependent velocity scale from (3.6),

$$
w \sim \bar{W}=-\frac{\bar{x}_{s}^{2}}{\mu} \frac{\partial P}{\partial z}\left(\frac{\bar{T}_{c}^{2}}{1+\bar{T}_{c}^{2}}\right),
$$




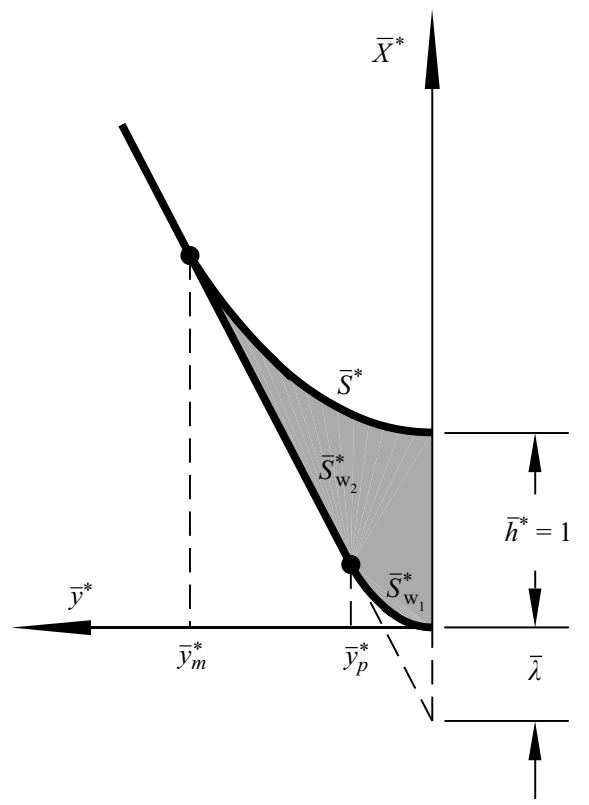

FiguRE 6. Dimensionless variables used in the cross-flow formulation (3.14-3.18). For any given axial position $z, \bar{h}^{*}=1$. The contact line is located at $\bar{y}^{*}=\bar{y}_{m}^{*}=f(1+\bar{\lambda}) \cos \alpha / \bar{T}_{c}$.

where the local length-scale ratio function is

$$
\bar{T}_{c} \equiv \frac{\bar{y}_{s}}{\bar{x}_{s}}=\tan \alpha+f \delta \bar{\lambda}
$$

where

$$
\bar{\lambda} \equiv \frac{\lambda}{(1-\lambda) h^{*}}
$$

with $h^{*}=h /\left(H-H_{c}\right)$. Note that under the present local-scaling definitions $\bar{h}^{*} \equiv h^{*} /$ $\left[h /\left(H-H_{c}\right)\right]=h / h=1$ as depicted in figure 6. The dimensional equation (3.6) nondimensionalized by $\bar{x}_{s}, \bar{y}_{s}$, and $\bar{W}$ becomes the equation

$$
1=\left(\frac{\bar{T}_{c}^{2}}{1+\bar{T}_{c}^{2}}\right) \frac{\partial^{2} \bar{w}^{*}}{\partial \bar{x}^{* 2}}+\left(\frac{1}{1+\bar{T}_{c}^{2}}\right) \frac{\partial^{2} \bar{w}^{*}}{\partial \bar{y}^{* 2}}+O\left(\epsilon_{c}^{2}\right),
$$

which when solved in the half-domain shown in figure 6 is subject to the contact-angle condition at the contact line, and boundary conditions as follows.

(i) The condition of no slip on the walls,

$$
\left.\begin{array}{lll}
\bar{w}^{*}=0 & \text { on } \bar{x}^{*}=\bar{S}_{\mathrm{W}_{1}}^{*}\left(\bar{y}^{*}\right), \quad 0 \leqslant \bar{y}^{*} \leqslant \bar{y}_{p}^{*}, \\
\bar{w}^{*}=0 & \text { on } \bar{x}^{*}=\bar{S}_{\mathrm{w}_{2}}^{*}\left(\bar{y}^{*}\right), \quad \bar{y}_{p}^{*}<\bar{y}^{*} \leqslant \bar{y}_{m}^{*}
\end{array}\right\}
$$

where $\bar{S}_{\mathrm{w}_{1}^{*}}$ and $\bar{S}_{\mathrm{w}_{2}^{*}}$ are the respective curved and straight portions of the wall as indicated in figure 6 and described by

$$
\bar{S}_{\mathrm{w}_{1}}^{*}=f \bar{\lambda}\left(1-\sqrt{1-\left(\frac{\bar{T}_{c} \bar{y}^{*}}{f \bar{\lambda}}\right)^{2}}\right) \text { and } \bar{S}_{\mathrm{w}_{2}}^{*}=\frac{\bar{T}_{c}}{\tan \alpha} \bar{y}^{*}-\bar{\lambda} .
$$


Note that at the point $\left(\bar{x}_{p}^{*}, \bar{y}_{p}^{*}\right), \bar{S}_{\mathrm{w}_{1}}^{*}=\bar{S}_{\mathrm{w}_{2}}^{*}$, and that $\bar{y}_{p}^{*}=f \bar{\lambda} \cos \alpha / \bar{T}_{c}$ and $\bar{y}_{m}^{*}=$ $f(1+\bar{\lambda}) \cos \alpha / \bar{T}_{c}$.

(ii) The symmetry condition

$$
\frac{\partial \bar{w}^{*}}{\partial \bar{y}^{*}}=0 \quad \text { on } \bar{y}^{*}=0
$$

(iii) The zero-shear-stress condition on the free surface:

$$
\frac{\partial \bar{w}^{*}}{\partial \bar{x}^{*}}-\frac{1}{\bar{T}_{c}^{2}} \frac{\partial \bar{S}^{*}}{\partial \bar{y}^{*}} \frac{\partial \bar{w}^{*}}{\partial \bar{y}^{*}}=0 \quad \text { on } \bar{x}^{*}=\bar{S}^{*}\left(\bar{y}^{*}\right) .
$$

The fluid surface $\bar{S}^{*}$ is stretched by the scaling and is a portion of an ellipse in the $\left(\bar{x}^{*}, \bar{y}^{*}\right)$-plane. The effective contact angle in this plane is given by

$$
\bar{\theta}=\arctan \left(\frac{\bar{T}_{c}(\tan (\alpha+\theta)-\tan \alpha)}{\bar{T}_{c}^{2}+\tan (\alpha+\theta) \tan \alpha}\right),
$$

where it is observed that when $\theta=0, \bar{\theta}=0$. All dimensionless quantities for the local problem are listed in table 2.

The local system of (3.14)-(3.18) is controlled by the parameters $\alpha$ and $\bar{\lambda}$ when $\theta=0$ and may be solved for $\bar{w}^{*}\left(\bar{x}^{*}, \bar{y}^{*}\right)$ and then integrated over the cross-section to determine the local dimensionless $z$-dependent average velocity:

$$
\langle\bar{w}\rangle^{*}=\frac{1}{\bar{A}^{*}} \iint \bar{w}^{*} \mathrm{~d} \bar{x}^{*} \mathrm{~d} \bar{y}^{*},
$$

where

$$
\bar{A}^{*} \equiv \frac{A}{\bar{x}_{s} \bar{y}_{s}}=\frac{F_{A}}{\bar{T}_{c}}(1+2 \bar{\lambda}),
$$

and

$$
F_{A}=f^{2}\left(\frac{\cos \theta \sin \delta}{\sin \alpha}-\delta\right) \sim \tan \alpha .
$$

For all values of $\alpha$ and $\theta, \tan \alpha \leqslant F_{A} \leqslant(4 / 3) \tan \alpha$. Asymptotic solutions for $\langle\bar{w}\rangle^{*}$ from (3.14)-(3.20) can be obtained under several limiting geometric conditions (i.e small and large $\alpha$, small and large $\bar{\lambda}$ ). Numerical values for $\langle\bar{w}\rangle^{*}$ are also readily computed. Such solutions will be outlined briefly herein; the discussion is facilitated by a preliminary description of the conversion method from the local dimensionless average velocity $\langle\bar{w}\rangle^{*}$ to the global one $\langle w\rangle^{*}$. This should provide a clearer understanding of the motivation for dwelling so much on the local cross-flow problem.

\subsubsection{Global scaling}

It will be shown that $\langle\bar{w}\rangle^{*}$ from (3.20) is a confined function $0.117 \leqslant\langle\bar{w}\rangle^{*} \leqslant 0.229$ for all values of $h, H_{c}$, and $\alpha$ for $\theta=0$. Furthermore, it will be shown that, with a priori knowledge of $\alpha$ and $\lambda,\langle\bar{w}\rangle^{*}$ may be treated as an $O(1)$ constant, incurring errors typically $\lesssim 5 \%$. Thus, the local scaling (see table 2 ) effectively captures most of the functional dependence of the cross-flow geometry for the rounded-corner problem. This being the case, a conversion from the local solution using the local $z$-dependent scaling to the global problem using a readily identified global scaling is possible. This step is necessary because the global length scales are known and constant whereas the local length scales are unknown and $z$-dependent. 


\begin{tabular}{lll}
\hline \multicolumn{1}{c}{ Lengths } & \multicolumn{1}{c}{ Velocities } & \multicolumn{1}{c}{ Other } \\
$x^{*}=x /\left(H-H_{c}\right)$ & $u^{*}=u / \epsilon_{c} W$ & $(\partial P / \partial z)^{*}=H f L(\partial P / \partial z) /(\sigma(1-\lambda))$ \\
$y^{*}=y /\left(\left(H-H_{c}\right) T_{c}\right)$ & $v^{*}=v / \epsilon_{c} W T_{c}$ & $t^{*}=W t / L$ \\
$z^{*}=\bar{z}^{*}=z / L$ & $w^{*}=w / W$ & $A^{*}=A /\left(\left(H-H_{c}\right)^{2} T_{c}\right)$ \\
$S^{*}=S /\left(H-H_{c}\right)$ & $\langle w\rangle^{*}=\langle w\rangle / W$ & $\dot{\mathrm{Q}}^{*}=\dot{\mathrm{Q}} /\left(W\left(H-H_{c}\right)^{2} T_{c}\right)$ \\
$h^{*}=h /\left(H-H_{c}\right)$ & $W=(1-\lambda)^{2} \epsilon_{c} \sigma T_{c}^{2} /\left(\mu f\left(1+T_{c}^{2}\right)\right)$ & $\lambda \equiv H_{c} / H, T=\tan \alpha /(1-\lambda)$ \\
$\mathscr{L}^{*}=\mathscr{L} / L$ & & $T_{c}=\tan \alpha+f \delta \lambda /(1-\lambda)$
\end{tabular}

TABLE 3. Global non-dimensionalized dependent and independent variables.

Using similar arguments to those for the local scaling, the global cross-flow length scales

$$
x \sim H-H_{c} \equiv x_{s}
$$

and

$$
y \sim\left(H-H_{c}\right) \tan \alpha+H_{c} f \delta \equiv y_{s}
$$

are chosen, they are listed with others in table 3 for the global problem. Applying these scales, (3.6) may be used to compute the global velocity scale

$$
w \sim W=\frac{x_{s}^{2}}{\mu}\left(\frac{\partial P}{\partial z}\right)_{s} \frac{T_{c}^{2}}{1+T_{c}^{2}},
$$

where

$$
\frac{\partial P}{\partial z} \sim\left(\frac{\partial P}{\partial z}\right)_{s} \equiv \frac{\sigma(1-\lambda)}{f H L}
$$

and $T_{c} \equiv y_{s} / x_{s}$.

The average velocity $\langle w\rangle$ non-dimensionalized by $W$ is the correct dimensionless velocity for the global mass balance (3.8), which may be expressed in terms of the local average velocity

$$
\langle w\rangle^{*} \equiv \frac{\langle w\rangle}{W}=\frac{\langle w\rangle}{\bar{W}} \frac{\bar{W}}{W}=\langle\bar{w}\rangle^{*} \frac{\bar{W}}{W} .
$$

As will be shown, $\langle\bar{w}\rangle^{*}$ from (3.20) is a weak $O(1)$ function that may be approximated as a constant. Thus, with $\bar{W}$ and $W$ known from (3.11) and (3.24), (3.26a) may be written as

$$
\langle w\rangle^{*}=-\langle\bar{w}\rangle^{*} \frac{\partial P}{\partial z}\left(\frac{\partial P}{\partial z}\right)_{s}^{-1} \frac{\bar{y}_{s}^{2}}{y_{s}^{2}} \frac{1+T_{c}^{2}}{1+T_{c}^{2}} .
$$

A ratio of pressure-gradient scales appears in this equation. Since the dimensional pressure in the fluid is given by $P=-\sigma / f\left(h+H_{c}\right)$, the pressure gradient is found to be $\partial P / \partial z=\left(\sigma / f\left(h+H_{c}\right)^{2}\right) \partial h / \partial z$. It is this local gradient that is scaled globally in (3.25). Subsequently, (3.26b) reduces to

$$
\langle w\rangle^{*}=-\frac{\langle\bar{w}\rangle^{*}}{k_{\lambda}^{2}}\left(\frac{1+k_{\delta} \bar{\lambda}}{1+\bar{\lambda}}\right)^{2} \frac{1+k_{\lambda}^{2} T^{2}}{1+\left(1+k_{\delta} \bar{\lambda}\right)^{2} \tan ^{2} \alpha} \frac{\partial h^{*}}{\partial z^{*}},
$$

where

$$
k_{\delta} \equiv f \delta / \tan \alpha
$$


and

$$
k_{\lambda} \equiv 1-\lambda+k_{\delta} \lambda
$$

For $\theta=0$, both $k_{\delta}(\alpha)$ and $k_{\lambda}(\alpha, \lambda)$ are weak $O(1)$ functions that may be treated in asymptotic analyses as $O(1)$ constants. For reference here,

$$
k_{\delta}(0)=\pi / 2 \leqslant k_{\delta} \leqslant 2=k_{\delta}(\pi / 2)
$$

and

$$
k_{\lambda}(\alpha, 0)=1 \leqslant k_{\lambda} \leqslant 2=k_{\lambda}(\pi / 2,1) .
$$

Note also that $k_{\lambda}(\alpha, 1)=k_{\delta}$.

The three most significant quantities observed in $(3.26 c)$ for the global problem are

$$
\tan \alpha, \quad T \equiv \frac{\tan \alpha}{1-\lambda}, \quad \text { and } \quad \bar{\lambda} .
$$

The latter two replace the more primitive parameters $\lambda$ and $h^{*}$ and are preferred for interrogation of the function multiplying the $\partial h^{*} / \partial z^{*}$ term in (3.26c) in subsequent asymptotic analysis. The quantities $\alpha$ and $\lambda$ are the sole parameters for the global problem. Note that for the sharp-corner case $\lambda=0$ and (3.26c) recovers the form of W\&L, where

$$
\langle w\rangle^{*}(\lambda=0) \equiv-F_{i} \frac{\partial h^{*}}{\partial z^{*}}=-\langle\bar{w}\rangle^{*} \frac{\partial h^{*}}{\partial z^{*}} .
$$

For a sharp corner, the local and global coordinates scale linearly with $h$ and $H$ respectively. Thus, $1 / 8 \lesssim F_{i}=\langle\bar{w}\rangle^{*} \leqslant 1 / 6$ for all $\alpha$ and $\theta$ when $H_{c}=0$. For a rounded corner, however, from $(3.26 c)$ it is seen that

$$
F_{i}=\langle\bar{w}\rangle^{*}\left(\frac{1+k_{\delta} \bar{\lambda}}{k_{\lambda}(1+\bar{\lambda})}\right)^{2} \frac{1+k_{\lambda}^{2} T^{2}}{1+\left(1+k_{\delta} \bar{\lambda}\right)^{2} \tan ^{2} \alpha} \equiv\langle\bar{w}\rangle^{*} I_{i} J_{i},
$$

which reveals an intricate $h^{*}$-dependence through $\bar{\lambda}$. With knowledge of the limits of $k_{\delta}$ and $k_{\lambda}$, inspection of (3.30) reveals that $I_{i}$ is also a fairly weak $O(1)$ function $1 \leqslant I_{i} \leqslant 4$ for all values of $h^{*}, \alpha$, and $\lambda$. This leaves $J_{i}$ as the primary function characterizing the $h^{*}$-dependence of the flow resistance for all $0 \leqslant J_{i} \leqslant 1$. The form $F_{i}=\langle\bar{w}\rangle^{*} I_{i} J_{i}$ substituted into (3.29) will be used to solve the non-dimensional version of the global mass balance (3.8). Before proceeding to this step, asymptotic and numerical solutions for $\langle\bar{w}\rangle^{*}$ will be briefly provided. The asymptotic solutions in particular are used to identify the various rounded-corner flow regimes as well as to provide the upper and lower bounds for $\langle\bar{w}\rangle^{*}$.

\subsection{Local average velocity $\langle\bar{w}\rangle^{*}$}

The local length-scale ratio parameter $\bar{T}_{c}$ for the dimensionless cross-flow problem (3.14) may be written as

$$
\bar{T}_{c} \equiv \bar{y}_{s} / \bar{x}_{s}=\left(1+k_{\delta} \bar{\lambda}\right) \tan \alpha .
$$

Asymptotic solution of the dimensionless system (3.14)-(3.20) is possible under limiting values of this parameter $\bar{T}_{c} \equiv \bar{T}_{c}(\alpha, \bar{\lambda})$. In general, the case $\bar{T}_{c}^{2} \ll 1$ is the narrowcorner limit and $\bar{T}_{c}^{2} \gg 1$ is the thin-film limit.

\subsubsection{Summary of asymptotic solutions for $\langle\bar{w}\rangle^{*}$}

For brevity, listed in table 4 are just the zeroth-order asymptotic solutions for $\langle\bar{w}\rangle^{*}$ for the various limiting geometric cases. Further details of the solutions are included in the Appendix. The five asymptotic limits are shown in figure 7. 
Limiting geometric conditions

$$
\left\langle\bar{w}_{0}\right\rangle^{*} \quad\left\langle\bar{w}_{0}\right\rangle_{h y d}^{*}
$$

Case I Narrow-corner limit, ${\overline{T_{c}}}^{2} \ll 1$

(a) narrow-sharp corner: $\alpha^{2} \ll 1, \bar{\lambda} \ll 1$

(b) narrow-corner rectangular section: $\alpha^{2} \bar{\lambda}^{2} \ll 1, \bar{\lambda}^{2} \gg 1$

$\begin{array}{ll}1 / 6 & 1 / 8 \\ 4 / 3 \pi^{2} & 2 / \pi^{2}\end{array}$

Case II Thin-film limit, ${\overline{T_{c}}}^{2} \gg 1$
(a) wide-sharp corner: $\Omega^{2} \ll 1, \bar{\lambda}^{2} \ll 1$
$1 / 7 \quad 1 / 18$
(b) wide-corner thin film: $\Omega^{2} \ll 1, \bar{\lambda}^{2} \gg 1$
$8 / 35$
$2 / 9$
(c) narrow-corner thin film: $\alpha^{2} \ll 1, \alpha^{2} \bar{\lambda}^{2} \gg 1$
$2 / 9$
$2 / \pi^{2}$

TABLE 4. Summary of zeroth-order asymptotic solutions for $\langle\bar{w}\rangle^{*}$.
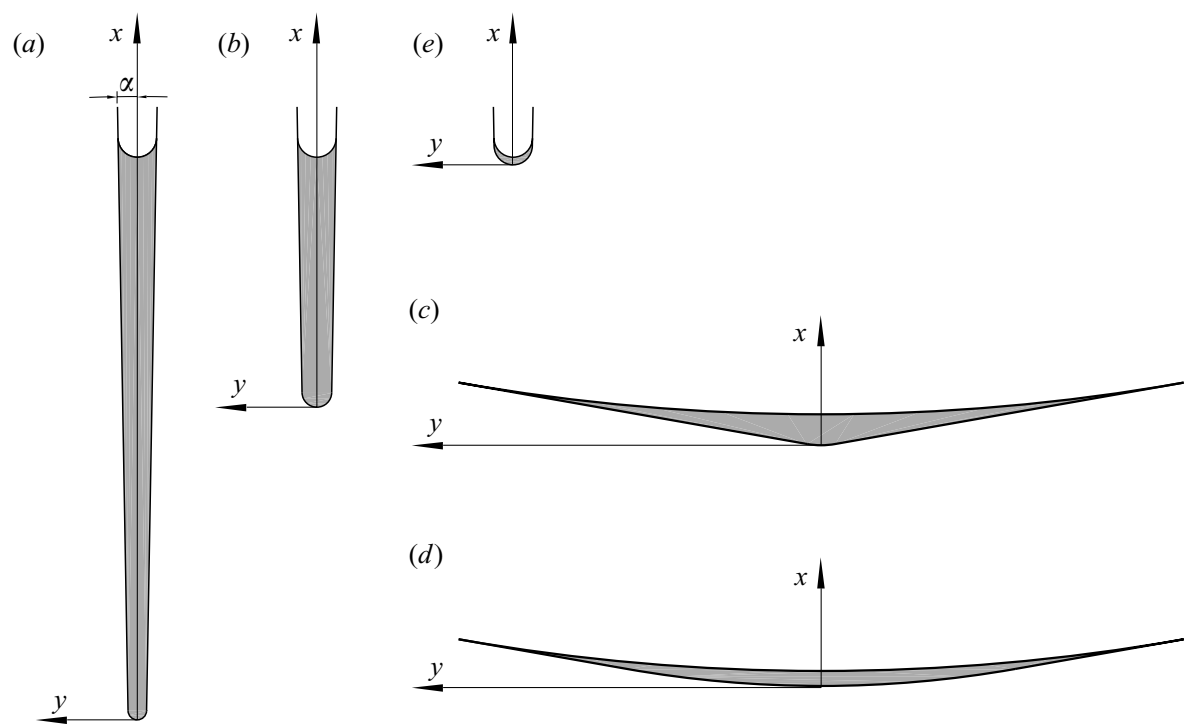

FIGURE 7. The geometric configurations for different limits: (a) narrow-sharp corner (case Ia), $(b)$ narrow-corner rectangular section $($ case $\mathrm{Ib}),(c)$ wide-sharp corner (case IIa), $(d)$ wide-corner thin film (case IIb), and (e) narrow-corner thin film (case IIc).

The slight-corner-roundedness results (Ia, IIa) listed in table 4 agree with the sharpcorner results of $\mathrm{W} \& \mathrm{~L}$. As observed in the table, the fact that $0.117 \lesssim\langle\bar{w}\rangle^{*} \lesssim 0.229$ for all cases argues that the general scaling approach should be used to provide an $O(1)$ banded function for the otherwise numerically determined coefficient $\langle\bar{w}\rangle^{*}$. For curiosity's sake, $\langle\bar{w}\rangle^{*}$ computed using the hydraulic diameter approach is also provided in table 4 for comparison. The range $1 / 18 \lesssim\langle\bar{w}\rangle_{h y d}^{*} \lesssim 2 / 9$ is significantly larger than that for $0.117 \leqslant\langle\bar{w}\rangle^{*} \leqslant 0.229$, which is itself larger than $1 / 8 \lesssim\langle\bar{w}\rangle^{*} \leqslant 1 / 6$ for the sharp corner (W\&L).

\subsubsection{Numerical solutions for $\langle\bar{w}\rangle^{*}$}

Numerical calculations were performed using Matlab with the PDE Toolbox, which implements a finite-element analysis on a triangular mesh. The meshing tool adaptively refines the mesh using the gradient of the solution. The refinement path consists of solving the PDE and refining the mesh until a specified number of elements (a minimum of 50000 in this study) has been reached. $\langle\bar{w}\rangle^{*}$ is computed for the range $0.000349 \leqslant \alpha \leqslant 1.567,0 \leqslant \lambda \leqslant 0.98$, and $0.02 \leqslant h^{*} \leqslant 1$ with $\theta=0$. The results of over 


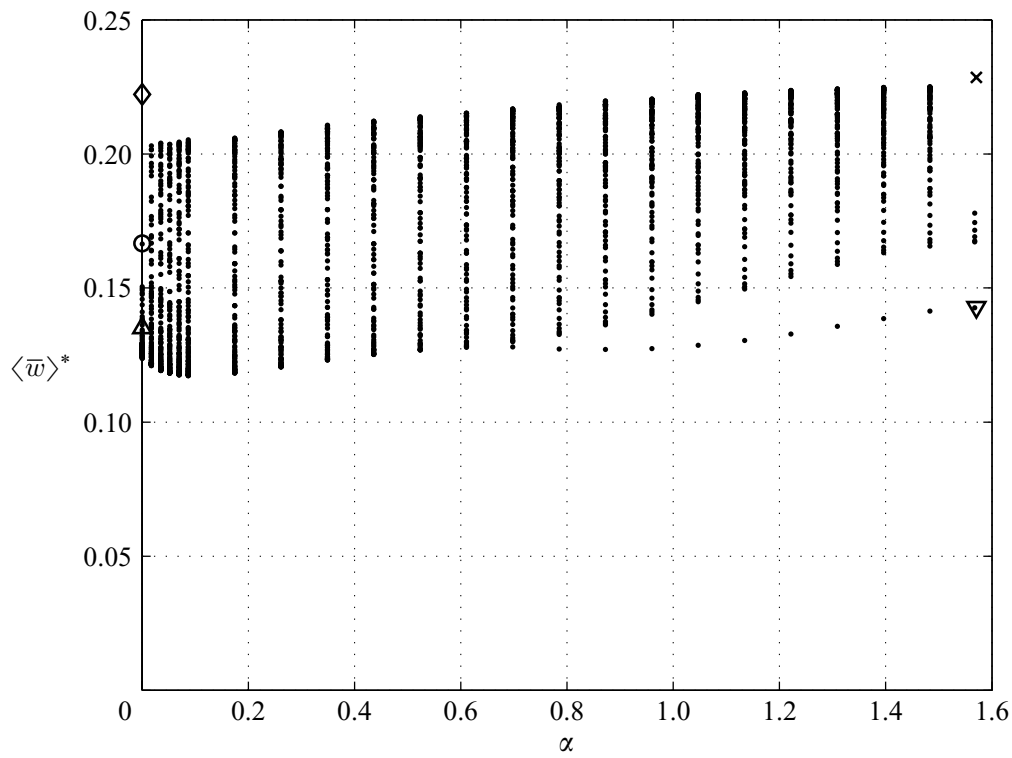

FiguRE 8. Local flow resistance $\langle\bar{w}\rangle^{*}$ as a function of $\alpha$ for various $h^{*}$ and $\lambda$. The dots are the numerical solutions; $\bigcirc$, narrow-sharp corner, $\alpha^{2} \ll 1$ and $\bar{\lambda} \ll 1$ (A 5); $\Delta$, narrow-corner rectangular section, $\alpha^{2} \bar{\lambda}^{2} \ll 1$ with $\bar{\lambda}^{2} \gg 1$ (A 6); $\nabla$, wide-sharp corner, $\Omega^{2} \ll 1$ and $\bar{\lambda}^{2} \ll 1$ (A 10); $\times$, wide-corner thin film, $\Omega^{2} \ll 1$ and $\bar{\lambda}^{2} \gg 1$ (A 11); $\diamond$, narrow-corner thin film, $\alpha^{2} \ll 1$ and $\bar{\lambda}^{2} \tan ^{2} \alpha \gg 1$ (A 16 ).

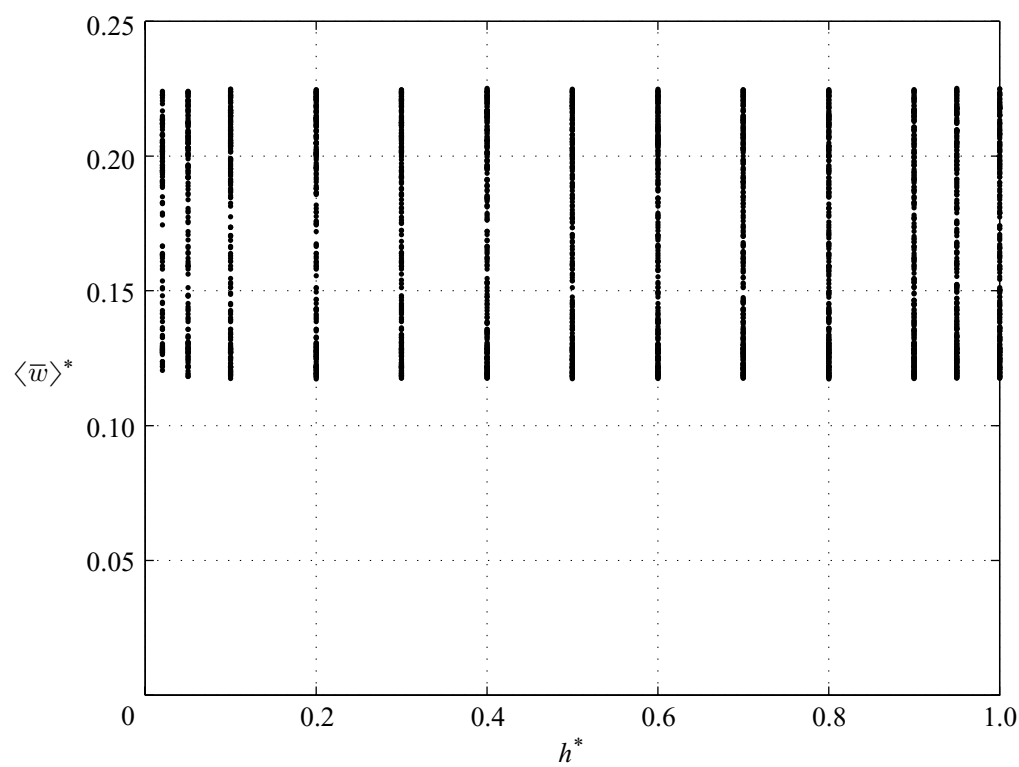

FIGURE 9. Local flow resistance $\langle\bar{w}\rangle^{*}$ as a function of $h^{*}$ for various $\alpha$ and $\lambda$.

3500 calculations are presented in figures 8 and 9 , where $\langle\bar{w}\rangle^{*}$ is plotted first as a function of $\alpha$ for various $h^{*}$ and $\lambda$ and then as a function of $h^{*}$ for various $\alpha$ and $\lambda$. The asymptotic values derived herein provide favourable benchmarks and are 

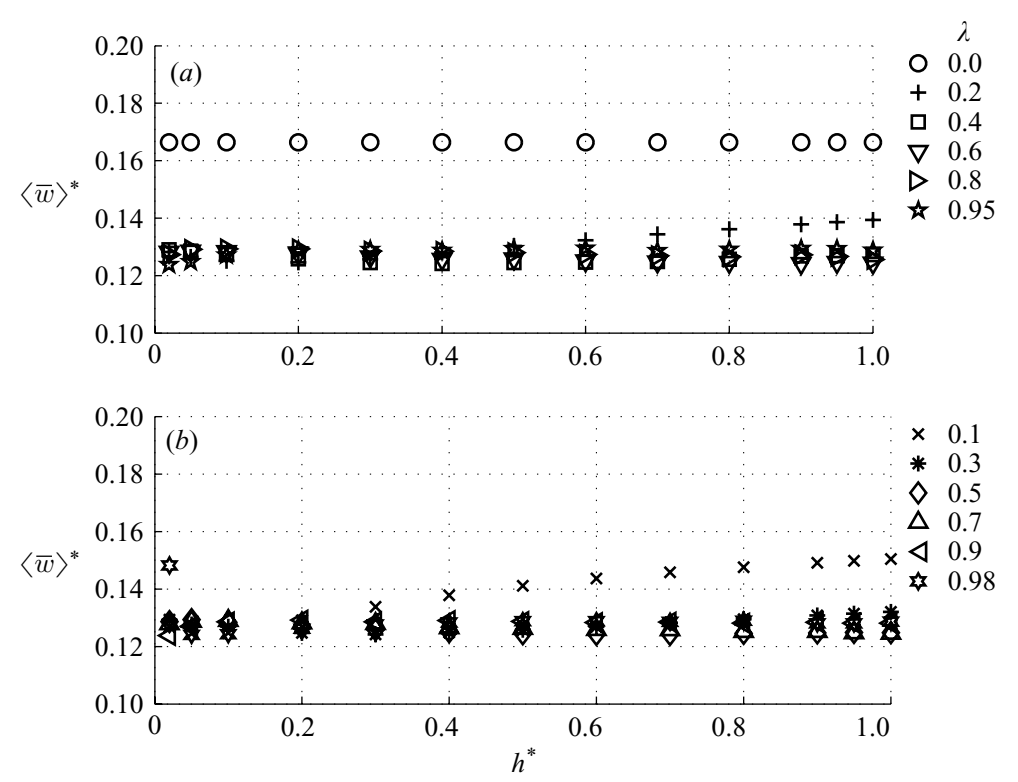

FIgURE 10. Local flow resistance $\langle\bar{w}\rangle^{*}$ as a function of $h^{*} ; \alpha=0.000349$.
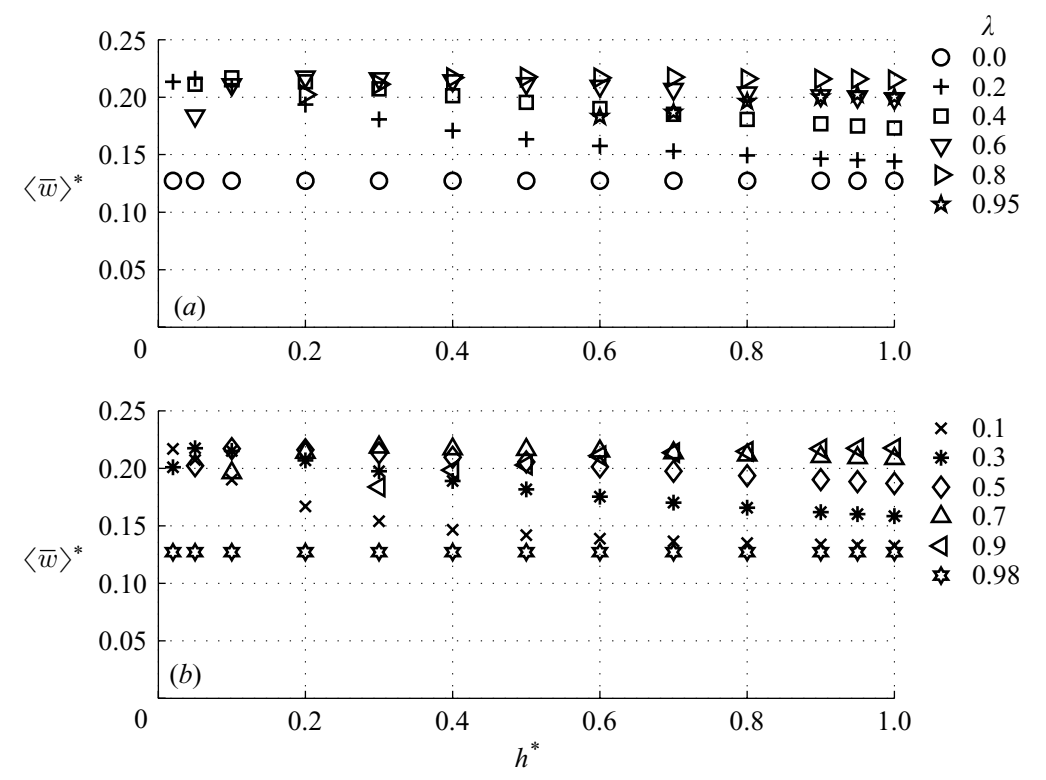

FIGURE 11. Local flow resistance $\langle\bar{w}\rangle^{*}$ as a function of $h^{*} ; \alpha=0.79$.

represented by large open symbols, while the numerical values are represented by small dots. It is clear that $0.117 \lesssim\langle\bar{w}\rangle^{*} \lesssim 0.229$ for all values of the parameters.

\subsubsection{Discussion of $\langle\bar{w}\rangle^{*}$ and $F_{i}$}

The numerical results of figure 9 are separated and replotted in figures 10-12. The corner half-angle $\alpha$ is fixed for each figure and calculations for fixed $\lambda$ are presented as functions of $h^{*}$. Several important observations may be made from these figures (i) The functional dependence of $\langle\bar{w}\rangle^{*}$ is intricate but weak. (ii) In 

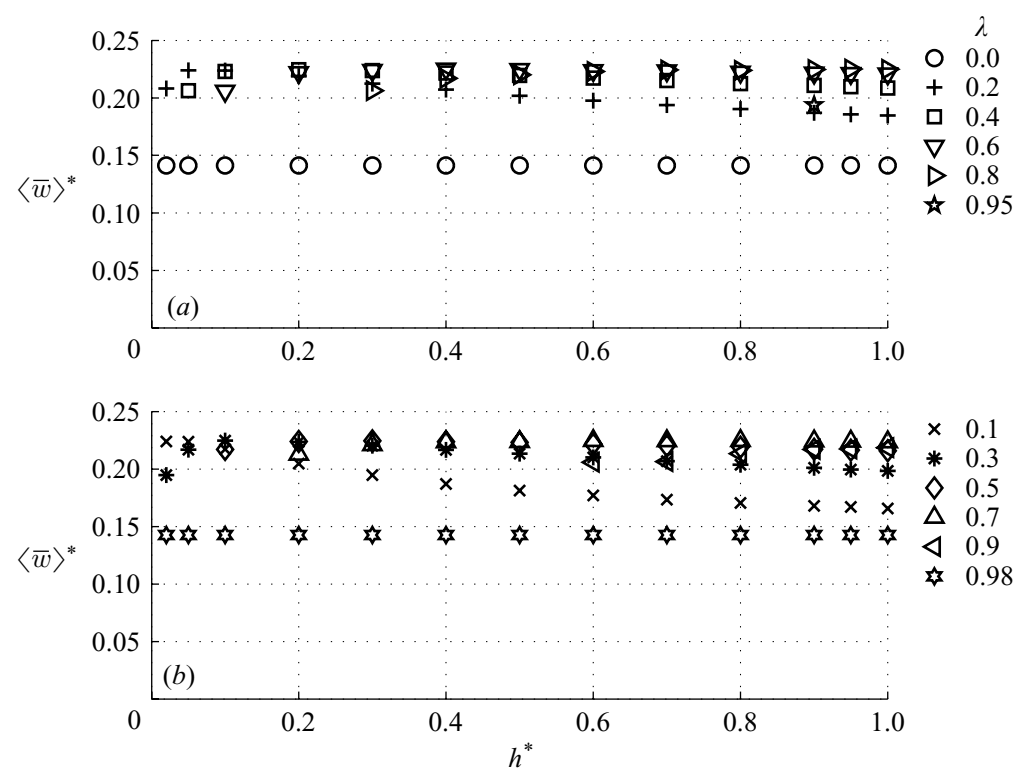

FIGURE 12. Local flow resistance $\langle\bar{w}\rangle^{*}$ as a function of $h^{*} ; \alpha=1.48$.

general, $\partial\langle\bar{w}\rangle^{*} / \partial h^{*} \ll 1$ except for $h^{*} \rightarrow 0$. (iii) However, the function $h^{*} \partial\langle\bar{w}\rangle^{*} / \partial h^{*} \rightarrow 0$ as $h^{*} \rightarrow 0$. (iv) $\partial\langle\bar{w}\rangle^{*} / \partial h^{*}$ can be either negative or positive. (v) For small values of $\alpha,\langle\bar{w}\rangle^{*}<\langle\bar{w}\rangle^{*}(\lambda=0)$; see figure 10. (vi) For large values of $\left.\alpha,\langle\bar{w}\rangle^{*}\right\rangle\langle\bar{w}\rangle^{*}(\lambda=0)$; see figures 11 and 12 .

The ideal situation would be to employ a scaling that renders $\langle\bar{w}\rangle^{*}$ a constant for all $\alpha, \lambda$, and $h^{*}$. This would imply that an exact solution to the cross-flow problem is obtained. Such a result is unlikely. Nonetheless, other local scalings were tried to this end such as $\bar{y}_{s}=h \tan \alpha+H_{c}$, which does not capture the crescent domain accurately as $\lambda \rightarrow 1$, and $\bar{y}_{s}=F_{A}\left(h+2 H_{c}\right) / 2$, which ensures that the dimensionless local section area $A^{*}=1$. Neither these nor other choices produced such narrowly confined results for $\langle\bar{w}\rangle^{*}$ as the present scaling, $\bar{y}_{s}=h \tan \alpha+H_{c} f \delta$.

The flow-resistance function $F_{i}$ given in (3.30) may be calculated using numerical values of $\langle\bar{w}\rangle^{*}$ for all values of the parameters. In practice, however, it is found that, for given $\alpha$ and $\lambda, F_{i}$ calculated using $\langle\bar{w}\rangle^{*}$ at $h^{*}=0.5$ is a very good and significantly simplifying approximation, as will be discussed later.

\section{Rounded-corner evolution equation}

Using the global scalings of table 3 and (3.26c), with $F_{i}$ given by (3.30), the mass balance (3.8) may be non-dimensionalized:

$$
\frac{\partial A}{\partial t}=-\frac{\partial}{\partial z}(A\langle w\rangle)=\frac{\partial}{\partial z}\left(A F_{i} \frac{\partial h}{\partial z}\right)=\frac{\partial}{\partial z}\left(A\langle\bar{w}\rangle^{*} I_{i} J_{i} \frac{\partial h}{\partial z}\right) .
$$

Unless otherwise specified, the '*' notation denoting dimensionless quantities is dropped for the remainder of the analysis with the exception of $\langle\bar{w}\rangle^{*}$.

Equation (4.1) can be rearranged into

$$
\frac{\partial A}{\partial t}=A\langle\bar{w}\rangle^{*} I_{i} J_{i}\left[\left(\frac{1}{A} \frac{\partial A}{\partial h}+\frac{1}{\langle\bar{w}\rangle^{*}} \frac{\partial\langle\bar{w}\rangle^{*}}{\partial h}+\frac{1}{I_{i}} \frac{\partial I_{i}}{\partial h}+\frac{1}{J_{i}} \frac{\partial J_{i}}{\partial h}\right)\left(\frac{\partial h}{\partial z}\right)^{2}+\frac{\partial^{2} h}{\partial z^{2}}\right],
$$


where

$$
\begin{gathered}
A=\left(\frac{F_{A}}{T_{c}}\right)(1+2 \bar{\lambda}) h^{2}, \\
I_{i}=\frac{1}{k_{\lambda}^{2}}\left(\frac{1+k_{\delta} \bar{\lambda}}{1+\bar{\lambda}}\right)^{2}, \\
J_{i}=\frac{1+k_{\lambda}^{2} T^{2}}{1+\left(1+k_{\delta} \bar{\lambda}\right)^{2} \tan ^{2} \alpha} .
\end{gathered}
$$

Applying the chain rule, we obtain $\partial A / \partial t=(\partial A / \partial h)(\partial h / \partial t), \partial I_{i} / \partial h=\left(\partial I_{i} / \partial A\right)$ $(\partial A / \partial h)$, etc. and, by factoring out the term $(1 / A)(\partial A / \partial h),(4.2)$ may be rewritten in terms primarily of $h$, through $\bar{\lambda}$, as

$$
\frac{\partial h}{\partial t}=\langle\bar{w}\rangle^{*} I_{i} J_{i}\left[\left(1+N_{\langle\bar{\psi}\rangle^{*}}+N_{I_{i}}+N_{J_{i}}\right)\left(\frac{\partial h}{\partial z}\right)^{2}+\left(\frac{1+2 \bar{\lambda}}{1+\bar{\lambda}}\right) \frac{h}{2} \frac{\partial^{2} h}{\partial z^{2}}\right],
$$

where

$$
\begin{gathered}
N_{\langle\bar{\psi}\rangle^{*}} \equiv \frac{h}{2\langle\bar{w}\rangle^{*}}\left(\frac{1+2 \bar{\lambda}}{1+\bar{\lambda}}\right) \frac{\partial\langle\bar{w}\rangle^{*}}{\partial h}, \\
N_{I_{i}} \equiv \frac{A}{I_{i}} \frac{\partial I_{i}}{\partial A}=-\frac{\bar{\lambda}\left(k_{\delta}-1\right)(1+2 \bar{\lambda})}{\left(1+k_{\delta} \bar{\lambda}\right)(1+\bar{\lambda})^{2}}, \\
N_{J_{i}} \equiv \frac{A}{J_{i}} \frac{\partial J_{i}}{\partial A}=k_{\delta} \bar{\lambda}\left(\frac{1+2 \bar{\lambda}}{1+\bar{\lambda}}\right) \frac{\left(1+k_{\delta} \bar{\lambda}\right) \tan ^{2} \alpha}{1+\left(1+k_{\delta} \bar{\lambda}\right)^{2} \tan ^{2} \alpha} .
\end{gathered}
$$

Equation (4.4) is the complete evolution equation for the dimensionless interface height $h$ for the rounded-corner problem. As emphasized above in the discussion (§3.2.3) of the numerical results for $\langle\bar{w}\rangle^{*}$, it can be demonstrated that the term $N_{\langle\bar{w}\rangle^{*}} \ll 1$ for all values of $h, \bar{\lambda}$, and $\alpha$ for $\theta=0$. The largest value of this term occurs for $h \sim O(1)$ with small but non-zero $\bar{\lambda}$ (note: $N_{\langle\bar{w}\rangle^{*}}=0$ for $\bar{\lambda}=0$ ). Under such conditions $\left|N_{\langle\bar{w}\rangle^{*}}\right| \lesssim 0.1$ is a maximum value. Also, observations made of the fairly weak function $1 \leqslant I_{i} \leqslant 4$ suggest that $N_{I_{i}}$ should also be a fairly weak function, which it is, taking a maximum value of $N_{I_{i}}=1 / 4$ when $\bar{\lambda}=1$ and $\alpha=\pi / 2$. In many cases $N_{I_{i}} \ll 1$ and, for $\bar{\lambda} \rightarrow 0$ and $\bar{\lambda} \rightarrow \infty, N_{I_{i}}=0$. (Though it is not ignored here, our approximate analysis may be further simplified by neglecting this term. For example, ignoring $N_{I_{i}}$ leads to errors for large $\lambda \lesssim \pm 4 \%$ and for small $\lambda$ at most $\pm 14 \%$.) Lastly, we have the range $0 \leqslant N_{J_{i}} \leqslant 2$ for all values of $h, \bar{\lambda}$, and $\alpha . N_{J_{i}} \rightarrow 0$ for $\bar{\lambda} \rightarrow 0$ (the sharp corner) and $N_{J_{i}} \rightarrow 2$ for $\bar{\lambda} \rightarrow \infty$ (the thin film).

\subsection{Simplified equation and regimes}

Neglecting $N_{\langle\bar{w}\rangle^{*}}$ and introducing a time scale $\tau=\langle\bar{w}\rangle^{*} t / 2$ that exploits the nearly constant nature of $\langle\bar{w}\rangle^{*},(4.4)$ reduces to

$$
\frac{\partial h}{\partial \tau}=I_{i} J_{i}\left[2\left(1+N_{I_{i}}+N_{J_{i}}\right)\left(\frac{\partial h}{\partial z}\right)^{2}+\left(\frac{1+2 \bar{\lambda}}{1+\bar{\lambda}}\right) h \frac{\partial^{2} h}{\partial z^{2}}\right] .
$$

This zeroth-order form of the evolution equation is represented below in three asymptotic regimes that can be identified by inspection of (4.6). 
(a) $\bar{\lambda} \ll 1$ This is the sharp-corner limit (figures $7 a$ and $7 c$ ), in which (4.6) simplifies to

$$
\frac{\partial h}{\partial \tau}=\frac{1}{k_{\lambda}^{2}} \frac{1+k_{\lambda}^{2} T^{2}}{1+\tan ^{2} \alpha}\left[2\left(\frac{\partial h}{\partial z}\right)^{2}+h \frac{\partial^{2} h}{\partial z^{2}}\right] \equiv C_{I}\left[2\left(\frac{\partial h}{\partial z}\right)^{2}+h \frac{\partial^{2} h}{\partial z^{2}}\right] .
$$

Regime I. For $\bar{\lambda} \ll 1, C_{I}=1$ and this equation further reduces to

$$
\frac{\partial h}{\partial \tau}=2\left(\frac{\partial h}{\partial z}\right)^{2}+h \frac{\partial^{2} h}{\partial z^{2}}
$$

as addressed in numerous previous investigations.

(b) $\bar{\lambda} \gg 1$ Under this constraint (4.6) reduces to

$$
\frac{\partial h}{\partial \tau}=2\left(\frac{k_{\delta}}{k_{\lambda}}\right)^{2} \frac{1+k_{\lambda}^{2} T^{2}}{1+k_{\delta}^{2} \bar{\lambda}^{2} \tan ^{2} \alpha}\left[\left(1+\frac{2 k_{\delta}^{2} \bar{\lambda}^{2} \tan ^{2} \alpha}{1+k_{\delta}^{2} \bar{\lambda}^{2} \tan ^{2} \alpha}\right)\left(\frac{\partial h}{\partial z}\right)^{2}+h \frac{\partial^{2} h}{\partial z^{2}}\right] .
$$

There are two limiting equations for such flows, as follows.

Regime II, narrow-corner rectangular section (figure $7 b$ ). For $\bar{\lambda} \gg 1$, when $\bar{\lambda}^{2} \alpha^{2} \ll 1$ is satisfied, the governing equation (4.9) simplifies to

$$
\frac{\partial h}{\partial \tau}=2\left(\frac{k_{\delta}}{k_{\lambda}}\right)^{2}\left[\left(\frac{\partial h}{\partial z}\right)^{2}+h \frac{\partial^{2} h}{\partial z^{2}}\right] \equiv C_{I I} \frac{\partial}{\partial z}\left(h \frac{\partial h}{\partial z}\right) .
$$

For $\lambda \rightarrow 1$, this equation reduces further to

$$
\frac{\partial h}{\partial \tau}=2 \frac{\partial}{\partial z}\left(h \frac{\partial h}{\partial z}\right) \text {. }
$$

Regime III, thin-film flow. When $\bar{\lambda}^{2} \tan ^{2} \alpha \gg 1$, (4.9) simplifies to

$$
\frac{\partial h}{\partial \tau}=\frac{2\left(1+k_{\lambda}^{2} T^{2}\right)}{k_{\lambda}^{2} \lambda^{2} T^{2}}\left[3 h^{2}\left(\frac{\partial h}{\partial z}\right)^{2}+h^{3} \frac{\partial^{2} h}{\partial z^{2}}\right] \equiv C_{I I I} \frac{\partial}{\partial z}\left(h^{3} \frac{\partial h}{\partial z}\right) .
$$

This is the thin-film limit of Darhuber, Troian \& Reisner (2001) (figures $7 d$ and 7e). When $T^{2} \gg 1$ and $\lambda \rightarrow 1, C_{I I I}=2$ and

$$
\frac{\partial h}{\partial \tau}=2 \frac{\partial}{\partial z}\left(h^{3} \frac{\partial h}{\partial z}\right) .
$$

Other specific limiting equations might be deduced from (4.6) but will vary only by coefficient and not by structure.

\subsection{Discussion}

It is important to note that $\bar{\lambda}$ is $h$-dependent and thus certain flows can possess widely varying values of $\bar{\lambda}$ as the fluid height $h$ changes along the corner. This is especially true for flows with an advancing front (or tip) where $h \rightarrow 0$. For example, for a narrow corner where $\alpha^{2} \ll 1$, it is possible for a flow to possess all three domains I, II, and III. This can be seen as one moves from the deepest portion of the fluid where $\bar{\lambda} \ll 1(\mathrm{I})$ to the advancing front where $\alpha^{2} \bar{\lambda}^{2} \gg 1$ (III). The narrow-corner rectangular domain requires $\bar{\lambda} \gg 1$ and $\alpha^{2} \bar{\lambda}^{2} \ll 1$ and is quite restrictive, making regime II rare in practice. Such flows do arise however for creeping capillary flows along thin rectangular slots that are slightly out of parallel. For advancing flows in rounded corners, flows can also transition from II to III or from I to III, or they can remain entirely in III. The non-overlapping regimes are separated by intermediate regions of the flow that are 
controlled by a known but more intricate nonlinear dependence on $h$ through $\bar{\lambda}$. For certain advancing flows, the streamwise curvature, ignored in this investigation, might eliminate the possibility of significant region III flow. Each flow regime has different response times and flow characteristics, several of which are considered here for a selection of flow scenarios.

For the various regimes, solutions are provided first for steady flows and sinusoidally perturbed flows. A similarity equation for imbibition (capillary rise) in capillaries with rounded interior corners is then derived and solved both asymptotically and numerically. The impact of the various terms in the governing evolution equation (4.6) that arise from corner roundedness are discussed in the light of this problem. The impact of the streamwise curvature is discussed for flows with advancing fronts where $h \rightarrow 0$ and analytic and numerical solutions for flows with finite 'tip' curvature modelling flows advancing over pre-existing columns of fluid are provided.

\subsection{Steady solutions}

The steady solutions to (4.6) are trivial in the various regimes and are listed here for both the dimensionless fluid height $h$ and the steady volumetric flow rate $\dot{Q}$ (see table 3 for the non-dimensionalization of $\dot{Q}$ ).

I. Sharp-corner flow, $\bar{\lambda} \ll 1(4.8)$

$$
\begin{gathered}
h=\left[1-\left(1-b^{3}\right) z\right]^{1 / 3}, \\
\dot{Q}_{I}=\frac{\lambda F_{A}}{3(1-\lambda) T_{c}}\langle\bar{w}\rangle^{*}\left(1-b^{3}\right) .
\end{gathered}
$$

II. Narrow-corner rectangular section, $\bar{\lambda} \gg 1, \bar{\lambda}^{2} \tan ^{2} \alpha \ll 1$ (4.11)

$$
\begin{gathered}
h=\left[1-\left(1-b^{2}\right) z\right]^{1 / 2}, \\
\dot{Q}_{I I}=\frac{\lambda F_{A}}{2(1-\lambda) T_{c}}\langle\bar{w}\rangle^{*}\left(1-b^{2}\right) .
\end{gathered}
$$

III. Thin-film flow, $\bar{\lambda} \gg 1, \bar{\lambda}^{2} \tan ^{2} \alpha \gg 1$ (4.13)

$$
\begin{gathered}
h=\left[1-\left(1-b^{4}\right) z\right]^{1 / 4}, \\
\dot{Q}_{I I I}=\frac{\lambda F_{A}}{4(1-\lambda) T_{c}}\langle\bar{w}\rangle^{*}\left(1-b^{4}\right) .
\end{gathered}
$$

The forms of the height $h$ and $\dot{Q}$ in $(4.14 a-4.16 b)$ assume known values of meniscus height at two locations, $h(z=0)=1$ and $h(z=1)=b$, within the same flow regime. These boundary conditions are equivalent to specifying a capillary-driven flow rate from $z=0$ to $z=1$. A maximum flow rate is achieved for $b=0$, which serves as an idealized model for complete fluid removal (sink) at the tip (i.e. suction). Other boundary conditions could have been specified. Steady flows that span two or more regimes, including any intermediate regimes, require more intricate steady-solution forms of the full equation

$$
\frac{\partial}{\partial z}\left(\frac{\sum_{m=0}^{3} N_{m} h^{m+3}}{\sum_{n=0}^{4} D_{n} h^{n}} \frac{\partial h}{\partial z}\right)=0,
$$

where the coefficients $N_{m}$ and $D_{n}$ are listed in table 5. This equation is readily solved numerically and a selection of results is presented in figure 13. In figure $13(a-c)$, pairs 


$$
\begin{array}{ll}
N_{0} & 2 k_{\delta}^{2} \lambda^{3} \\
N_{1} & k_{\delta}\left(4+k_{\delta}\right)(1-\lambda) \lambda^{2} \\
N_{2} & 2\left(1+k_{\delta}\right)(1-\lambda)^{2} \lambda \\
N_{3} & (1-\lambda)^{3} \\
D_{0} & k_{\delta}^{2} \lambda^{4} \tan ^{2} \alpha \\
D_{1} & 2 k_{\delta}\left(1+k_{\delta}\right)(1-\lambda) \lambda^{3} \tan ^{2} \alpha \\
D_{2} & \frac{1}{2}(1-\lambda)^{2} \lambda^{2}\left(2+4 k_{\delta}+k_{\delta}^{2}-k_{\delta}\left(4+k_{\delta}\right) \cos 2 \alpha\right) \sec ^{2} \alpha \\
D_{3} & (1-\lambda)^{3} \lambda\left(2+k_{\delta}-k_{\delta} \cos 2 \alpha\right) \sec ^{2} \alpha \\
D_{4} & (1-\lambda)^{4} \sec ^{2} \alpha
\end{array}
$$

TABLE 5. Coefficients of the steady-state flow equation.
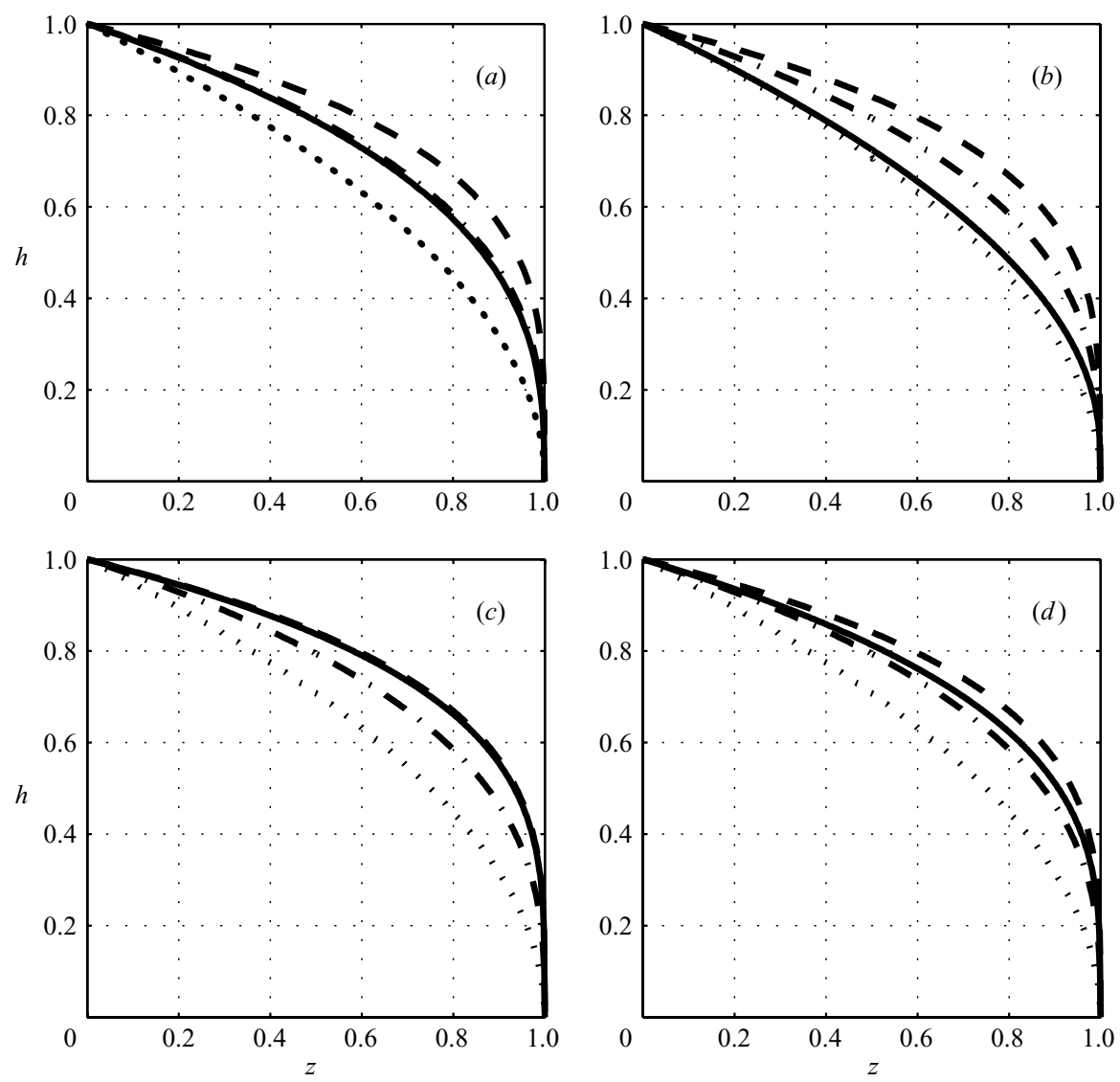

FiguRE 13. Steady-state solutions with $b=0$ for a selection of $\alpha$ and $\lambda$ conditions. $(a)$ predominantly sharp corner, $\alpha=\pi / 4, \lambda=0.05 ;(b)$ predominantly rectangular section, $\alpha=0.01, \lambda=0.9$; (c) predominantly thin film, $\alpha=1, \lambda=0.9 ;(d)$ regime intermediate between I and III, $\alpha=\pi / 4$, $\lambda=0.5$. Solid line, numerical solutions (4.17); dash dot line, sharp-corner regime (I) (4.14a); dotted line, rectangular-section regime (II) (4.15a); dashed line thin-film regime (III) (4.16a).

of $\alpha$ and $\lambda$ values are chosen in the neighbourhood of each of the three flow regimes: sharp-corner (figure 13a), rectangular-section (figure 13b), and thin-film (figure 13c). The respective exact solutions listed in $(4.14 a),(4.15 a)$, and $(4.16 a)$ provide the best 
approximations to the full numerical solution in these cases. In figure 13(d) typical intermediate values of $\alpha$ and $\lambda$ are chosen for which the numerical result falls between the sharp-corner and thin-film flow regimes. The analytical solutions are given by $h=(1-z)^{a}$, where $a=1 / 3,1 / 2$, and $1 / 4$ for the sharp-corner (I), rectangular-section (II), and thin-film (III) regimes, respectively.

\subsection{Infinite-column solutions}

For a sinusoidally perturbed liquid column in a rounded corner of infinite extent (Weislogel 2001b), $h=1$ defines the mean unperturbed height of the liquid. Introducing the expansion

$$
h=1+\epsilon_{c} h_{1}+\epsilon_{c}^{2} h_{2}+\ldots,
$$

solutions for the transient relaxation of the perturbed surface are readily obtained for the various regimes by substituting (4.18) into the corresponding equations (4.8), (4.11), or (4.13). It can be shown that to leading order

$$
\begin{aligned}
& \text { I. } h=1+\epsilon_{c} D_{1} \exp \left(-\xi^{2} \tau\right) \cos \left(\xi z+D_{2}\right)+O\left(\epsilon_{c}^{2}\right), \\
& \text { II and III. } h=1+\epsilon_{c} D_{3} \exp \left(-2 \xi^{2} \tau\right) \cos \left(\xi z+D_{4}\right)+O\left(\epsilon_{c}^{2}\right),
\end{aligned}
$$

where $\xi=\xi^{\prime} / L$ is the dimensionless wave number and $D_{1}$ through $D_{4}$ are constants to be determined by the boundary conditions. For all regimes the decaying exponents, after redimensionalization, reduce to

$$
\xi^{2} \tau \sim \frac{\langle\bar{w}\rangle^{*} \epsilon_{c}^{2}(1-\lambda)}{f} \frac{T_{c}^{2}}{1+T_{c}^{2}} \frac{\sigma t}{\mu H}=\epsilon_{c}^{2}\left(\mathscr{F} C a_{t}\right)^{-1}
$$

where $H$ is the unperturbed height of the interface, $\mathscr{F}=f\left(1+T_{c}^{2}\right) /\left(\langle\bar{w}\rangle^{*}(1-\lambda) T_{c}^{2}\right)$, and $C a_{t}=\mu H / \sigma t$ is a time-dependent capillary number.

Recalling that $\langle\bar{w}\rangle^{*}$ can be approximated as a constant, for given $H$ and fluid properties $\sigma / \mu$, the response time of the disturbance is dependent on a geometric time constant

$$
G T C=\frac{f}{(1-\lambda)^{3}} \frac{1+T_{c}^{2}}{T_{c}^{2}},
$$

which is plotted in figure 14 as a function of both $\alpha$ and $\lambda$. As can be seen from the figure, GTC increases with $\lambda$ for a fixed value of $\alpha$. The fastest response of the fluid occurs for the lowest value of $G T C$, which occurs at $\alpha=\pi / 6$ (30 in the figure) when $\lambda=0$, as was shown by Weislogel (2001b) for the sharp-corner problem. However, as $\lambda$ increases, the fastest response time occurs for increasingly lower values of $\alpha$. It appears that for $\lambda \rightarrow 1$, GTC is minimal for $\alpha \rightarrow 0$.

\subsection{Similarity solution for imbibition problem}

In this section a solution for spontaneously advancing flows in capillaries with rounded interior corners is investigated. This is the imbibition problem studied by Dong \& Chatzis (1995), or the capillary-rise problem of W\&L, where the advancing fluid rapidly achieves a constant height $H$ at the coordinate origin for the flow, as addressed in detail in Weislogel (2001a). Thus, $h(z=0, t=0)=1$ and $h\left(z_{\text {tip }}, t\right)=0$ are suitable boundary conditions for this problem. Under these conditions the general evolution equation (4.6) admits a power-law similarity transformation, giving rise to a large similarity equation appropriate for all parametric regimes. Asymptotic and numeric solutions to this equation are instructive concerning rounded-corner flow in 


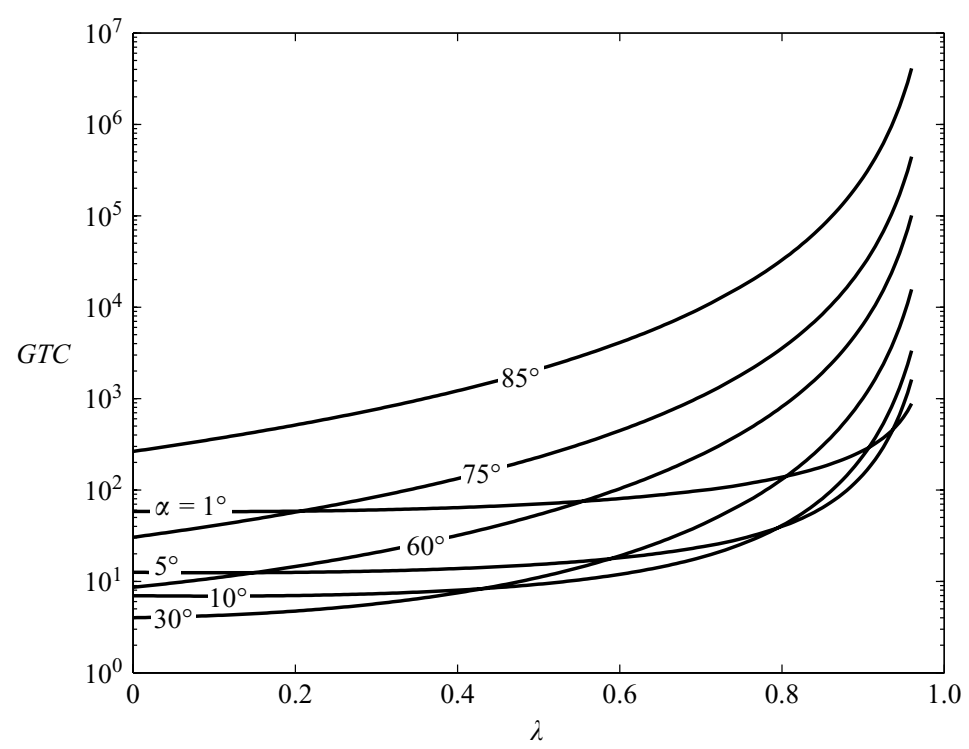

FIGURE 14. Geometric time constant $\operatorname{GTC}(\lambda ; \alpha)$.

general. They also permit the development of useful closed-form solutions for this fundamental problem.

Treating $\langle\bar{w}\rangle^{*}$ as constant, (4.6) is rearranged and rewritten here in terms of $h$ rather than $\bar{\lambda}$ :

$$
\begin{aligned}
& {[(1-\lambda) h+\lambda] \frac{\partial h}{\partial \tau}} \\
& \quad=\Lambda \frac{\partial}{\partial z}\left(\frac{\left[(1-\lambda) h+k_{\delta} \lambda\right]^{2}\left[(1-\lambda) h^{2}+2 \lambda h\right] h^{2}}{[(1-\lambda) h+\lambda]^{2}\left\{(1-\lambda)^{2} h^{2}+\left[(1-\lambda) h+k_{\delta} \lambda\right]^{2} \tan ^{2} \alpha\right\}} \frac{\partial h}{\partial z}\right),
\end{aligned}
$$

where

$$
\Lambda=\frac{(1-\lambda)^{2}+\left[(1-\lambda)+k_{\delta} \lambda\right]^{2} \tan ^{2} \alpha}{\left[(1-\lambda)+k_{\delta} \lambda\right]^{2}} .
$$

This is the form of a nonlinear diffusion equation, with polynomial functions of $h$ multiplying all derivatives after further rearrangement. Such an equation is more or less similar to a class of nonlinear diffusion equations of simpler form discussed by Fujita (1952), who showed the existence of similarity solutions for such equations. Despite the size and complexity of (4.22), the equation is self-similar under the same power-law transformation as the sharp-corner problem. Introducing

$$
h=F(\eta), \quad \eta=z /(2 \tau)^{1 / 2}, \quad \mathscr{L}_{\text {tip }}=\eta_{\text {tip }}(2 \tau)^{1 / 2},
$$

(4.22) transforms to a 23 -term similarity equation

$$
\sum_{m=0}^{6} Z Z_{m} F^{m+3} F_{\eta \eta}+\sum_{n=0}^{6} Z_{n} F^{n+2} F_{\eta}^{2}+\sum_{k=0}^{8} T_{k} F^{k} \eta F_{\eta}=0
$$

with coefficients $Z Z_{m}, Z_{n}$, and $T_{k}$ as listed in table 6. In (4.25) the subscript notation for the ordinary differentiation of $F(\eta)$ with respect to $\eta$ is employed - all other 
$Z Z_{0} \quad 2 k_{\delta}^{4} \lambda^{6} \tan ^{2} \alpha \Lambda_{\text {num }}$

$Z Z_{1} \quad k_{\delta}^{3}\left(8+3 k_{\delta}\right)(1-\lambda) \lambda^{5} \tan ^{2} \alpha \Lambda_{\text {num }}$

$Z Z_{2} \quad k_{\delta}^{2}(1-\lambda)^{2} \lambda^{4}\left[2+\left(12+12 k_{\delta}+k_{\delta}^{2}\right) \tan ^{2} \alpha\right] \Lambda_{\text {num }}$

$Z Z_{3} \quad k_{\delta}(1-\lambda)^{3} \lambda^{3}\left[4+3 k_{\delta}+2\left(4+9 k_{\delta}+2 k_{\delta}^{2}\right) \tan ^{2} \alpha\right] \Lambda_{\text {num }}$

$Z Z_{4} \quad(1-\lambda)^{4} \lambda^{2}\left[2+6 k_{\delta}+k_{\delta}^{2}+2\left(1+6 k_{\delta}+3 k_{\delta}^{2}\right) \tan ^{2} \alpha\right] \Lambda_{\text {num }}$

$Z Z_{5} \quad(1-\lambda)^{5} \lambda\left[3\left(1+k_{\delta}\right)-k_{\delta} \cos 2 \alpha\right] \sec ^{2} \alpha \Lambda_{\text {num }}$

$Z Z_{6} \quad(1-\lambda)^{6} \sec ^{2} \alpha \Lambda_{\text {num }}$

$Z_{0} \quad 6 k_{\delta}^{4} \lambda^{6} \tan ^{2} \alpha \Lambda_{\text {num }}$

$Z_{1} \quad 6 k_{\delta}^{3}\left(4+k_{\delta}\right)(1-\lambda) \lambda^{5} \tan ^{2} \alpha \Lambda_{\text {num }}$

$Z_{2} \quad 2 k_{\delta}^{2}(1-\lambda)^{2} \lambda^{4}\left[1+\left(18+12 k_{\delta}+k_{\delta}^{2}\right) \tan ^{2} \alpha\right] \Lambda_{\text {пит }}$

$Z_{3} \quad 4 k_{\delta}(1-\lambda)^{3} \lambda^{3}\left[2+\left(6+9 k_{\delta}+2 k_{\delta}^{2}\right) \tan ^{2} \alpha\right] \Lambda_{\text {пит }}$

$Z_{4} \quad 6(1-\lambda)^{4} \lambda^{2}\left[1+k_{\delta}+\left(1+4 k_{\delta}+2 k_{\delta}^{2}\right) \tan ^{2} \alpha\right] \Lambda_{\text {num }}$

$Z_{5} \quad(1-\lambda)^{5} \lambda\left(6+5 k_{\delta}-3 k_{\delta} \cos 2 \alpha\right) \sec ^{2} \alpha \Lambda_{\text {num }}$

$Z_{6} \quad 2(1-\lambda)^{6} \sec ^{2} \alpha \Lambda_{\text {numy }}$

$T_{0} \quad k_{\delta}^{4} \lambda^{8} \tan ^{4} \alpha \Lambda_{\text {den }}$

$T_{1} \quad 4 k_{\delta}^{3}\left(1+k_{\delta}\right)(1-\lambda) \lambda^{7} \tan ^{4} \alpha \Lambda_{\text {den }}$

$T_{2} \quad k_{\delta}^{2}(1-\lambda)^{2} \lambda^{6}\left[4+8 k_{\delta}+3 k_{\delta}^{2}-\left(2+8 k_{\delta}+3 k_{\delta}^{2}\right) \cos 2 \alpha\right] \sec ^{2} \alpha \tan ^{2} \alpha \Lambda_{d e n}$

$T_{3} \quad 2 k_{\delta}(1-\lambda)^{3} \lambda^{5}\left[2+8 k_{\delta}+6 k_{\delta}^{2}+k_{\delta}^{3}-k_{\delta}\left(4+6 k_{\delta}+k_{\delta}^{2}\right) \cos 2 \alpha\right] \sec ^{2} \alpha \tan ^{2} \alpha \Lambda_{d e n}$

$T_{4} \quad(1-\lambda)^{4} \lambda^{4}\left[1+2\left(1+8 k_{\delta}+6 k_{\delta}^{2}\right) \tan ^{2} \alpha+\left(1+16 k_{\delta}+36 k_{\delta}^{2}+16 k_{\delta}^{3}+k_{\delta}^{4}\right) \tan ^{4} \alpha\right] \Lambda_{d e n}$

$T_{5} \quad 4(1-\lambda)^{5} \lambda^{3}\left[1+2\left(1+3 k_{\delta}+k_{\delta}^{2}\right) \tan ^{2} \alpha+\left(1+6 k_{\delta}+6 k_{\delta}^{2}+k_{\delta}^{3}\right) \tan ^{4} \alpha\right] \Lambda_{d e n}$

$T_{6} \quad(1-\lambda)^{6} \lambda^{2}\left[12+16 k_{\delta}+5 k_{\delta}^{2}-2 k_{\delta}\left(8+3 k_{\delta}\right) \cos 2 \alpha+k_{\delta}^{2} \cos 4 \alpha\right] \sec ^{4} \alpha \Lambda_{\text {den }} / 2$

$T_{7} \quad 2(1-\lambda)^{7} \lambda\left(2+k_{\delta}-k_{\delta} \cos 2 \alpha\right) \sec ^{4} \alpha \Lambda_{d e n}$

$T_{8} \quad(1-\lambda)^{8} \sec ^{4} \alpha \Lambda_{\text {den }}$

TABLE 6. Coefficients of the full similarity equation (4.25); note that $\Lambda=\Lambda_{\text {num }} / \Lambda_{\text {den }}$ from (4.23), where 'num' and 'den' denote the numerator and denominator, respectively.

sub- or superscripts are indices. The boundary conditions for (4.25) are

$$
F(0)=1, \quad F\left(\eta_{\text {tip }}\right)=0,
$$

and the integral volume constraint

$$
\left.F_{\eta}\right|_{\eta=0}=-\frac{1}{2(1+\lambda)} \int_{0}^{\eta_{t i p}}\left[(1-\lambda) F^{2}+2 \lambda F\right] \mathrm{d} \eta
$$

required to close the system - owing to the introduction of the unknown advancing front $\eta_{t i p}$. In addition, the value of $F_{\eta}\left(\eta_{t i p}\right)$ can be determined from the similarity equation, obtaining

$$
F_{\eta}\left(\eta_{t i p}\right)= \begin{cases}-\eta_{t i p} / 2 & \text { if } \lambda=0, \\ -(2-2 \lambda+\pi \lambda)^{2} \eta_{t i p} / 2 \pi^{2} & \text { if } \alpha=0 \text { and } \lambda>0, \\ -\infty & \text { otherwise. }\end{cases}
$$

Dramatic simplification of the governing similarity equation results in the three asymptotic regimes, which correspondingly reduce (4.25) as follows. 


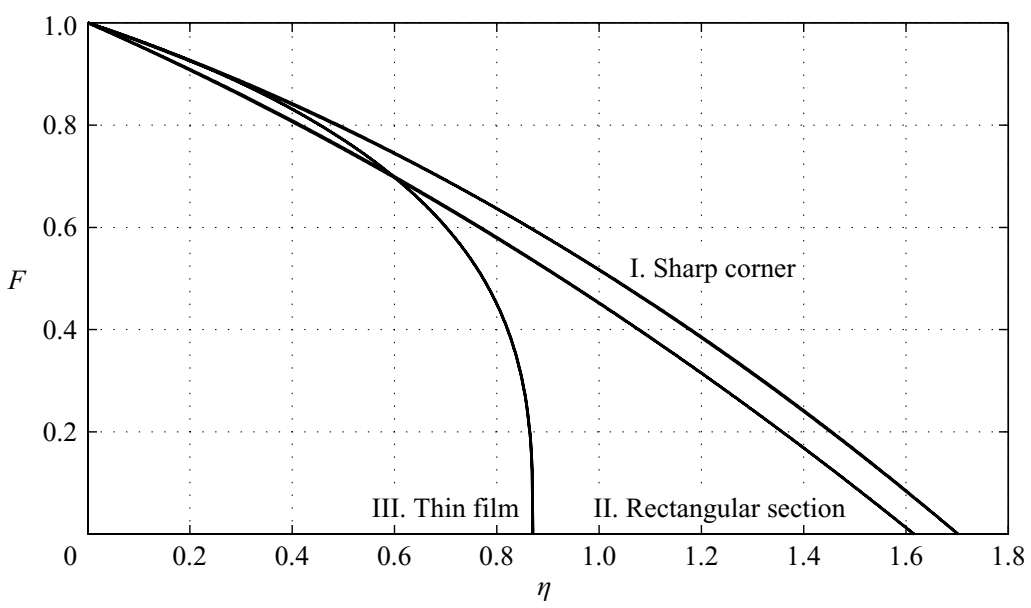

FIGURE 15. Numerical and approximate analytic solutions for three asymptotic flow regimes; for each case, the difference between the numerical and approximate analytic data is indistinguishable.

\section{Sharp-corner flow}

$$
0=\eta F_{\eta}+2 F_{\eta}^{2}+F F_{\eta \eta} .
$$

II. Narrow-corner rectangular section

$$
0=\eta F_{\eta}+2\left(F F_{\eta}\right)_{\eta} .
$$

III. Thin-film flow

$$
0=\eta F_{\eta}+2\left(F^{3} F_{\eta}\right)_{\eta} .
$$

The sharp-corner-flow asymptotic equation has been derived and solved by numerous authors (e.g. Lenormand \& Zarcone 1984; Dong \& Chatzis 1995; Romero \& Yost 1996; Weislogel \& Lichter 1998). The thin-film flow equation was also derived by Darhuber et al. (2001), who studied the capillary flow along flat hydrophilic microstripes. It will be shown that approximate analytic or exact numerical solutions to the limiting similarity equations $(4.29 a-c)$ provide envelopes for numerical solutions of the full equation (4.25). Approximate analytic solutions to $(4.29 a-c)$ can be obtained by the method of Mayer, McGrath \& Steele (1983) using polynomial trial functions. The results of this method are presented here:

$$
\begin{gathered}
F_{I}=1-0.571 \eta^{*}-0.429 \eta^{* 2}, \\
F_{I I}=1-0.698 \eta^{*}-0.302 \eta^{* 2}, \\
F_{I I I}=\left(1-0.865 \eta^{*}-0.135 \eta^{* 2}\right)^{1 / 3},
\end{gathered}
$$

where $\eta^{*}=\eta / \eta_{\text {tip }}$. These solutions compare well $\left(<1.5 \%\right.$ for $F$ and $<3.9 \%$ for $\left.F_{\eta}(0)\right)$ with the numerical solutions in the respective regimes, as shown in figure 15 and table 7.

Equation (4.25) and the associated boundary conditions were also solved numerically for $F(\eta)$ and representative results are presented in figure 16 for a variety of $\lambda$ values for $\alpha=\pi / 4$ with $\theta=0$. The enveloping solutions of regime I (4.29a) and regime III $(4.29 c)$ are given in the figure. Note that the narrow-corner rectangular section regime (II) does not exist for this flow since the condition $\alpha^{2} \ll 1$ is not satisfied 
Regime

Analytical

Similarity

$\begin{array}{lcllll} & \eta_{\text {tip }} & F_{\eta}(0) & F_{\eta}\left(\eta_{\text {tip }}\right) & F_{\eta}(0) & F_{\eta}\left(\eta_{\text {tip }}\right) \\ \text { I. Sharp corner } & 1.702 & -0.335 & -0.84 & -0.349 & -0.851 \\ \text { II. Rectangular section } & 1.616 & -0.432 & -0.806 & -0.444 & -0.808 \\ \text { III. Thin film } & 0.871 & -0.331 & -\infty & -0.333 & -\infty\end{array}$

TABLE 7. Approximate analytic and numerical similarity solution results for the three regimes.

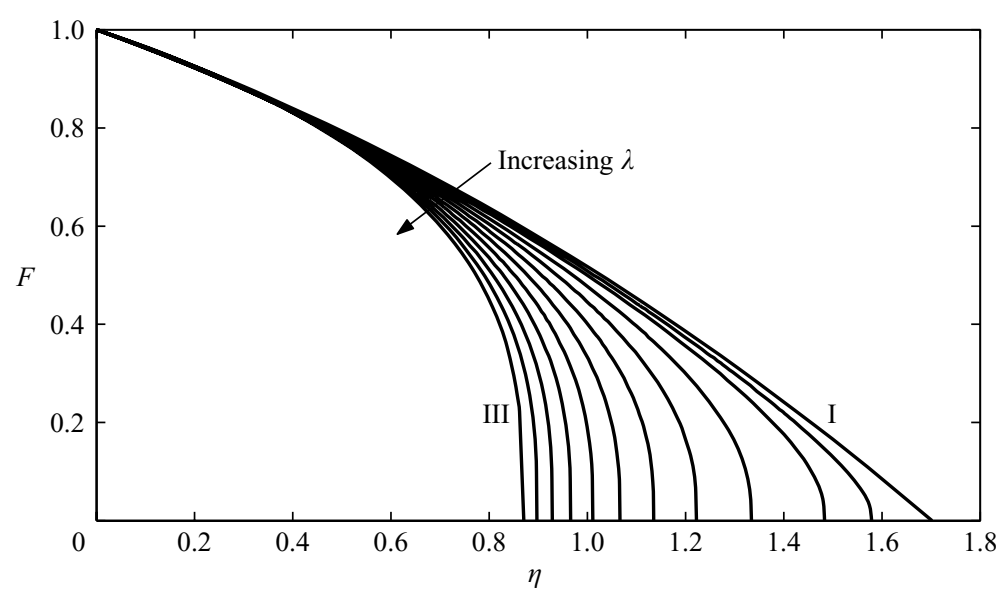

FIGURE 16. Similarity solutions for $\lambda=0,0.05,0.1,0.2, \ldots, 1.0$ with $\alpha=\pi / 4$.

for $\alpha=\pi / 4$. If the flow is deep enough $(\bar{\lambda} \ll 1)$, there is a transition for this flow from regime $\mathrm{I}$ to an intermediate regime and then to regime III. Figure 16 clearly shows quantitatively how the flow is 'retarded' (decreasing $\eta_{\text {tip }}$ ) for increasing corner roundedness $\lambda$. It is also interesting to note that $F_{\eta}(0)$ is not nearly as dependent on $\lambda$ as is $\eta_{\text {tip }}$.

Figure 16 also reveals that the slope of the free surface becomes increasingly steep as the advancing tip is approached. In fact, for regime III, $F_{\eta} \rightarrow-\infty$ as $\eta \rightarrow \eta_{t i p}$, whereas for regimes I and II $F_{\eta}\left(\eta_{t i p}\right)=-\eta_{t i p} / 2$. For gradients too steep at the tip, the assumptions of a locally slender column $\epsilon_{c}^{2} \ll 1$ and dominant cross-stream curvature $\epsilon_{c}^{2} f \ll 1$ may no longer be satisfied and the solutions are expected to break down; this was addressed by Darhuber et al. (2001). The slender-column and dominant cross-stream curvature constraints may be written in local differential form as

$$
\epsilon_{c}^{2} \sim \frac{\epsilon_{c}^{2} F_{\eta}^{2}}{2 \tau} \ll 1
$$

and

$$
\epsilon_{c}^{2} f \sim \epsilon_{c}^{2} f\left(F+\frac{\lambda}{1-\lambda}\right) \frac{F_{\eta \eta}}{2 \tau} \ll 1,
$$

respectively. Further manipulation reduces $(4.31 a)$ to

$$
F_{\eta}^{2} \ll \frac{\sigma}{\mu} \frac{1-\lambda}{f} \frac{\langle\bar{w}\rangle^{*}}{H} \frac{T_{c}^{2}}{1+T_{c}^{2}} t
$$




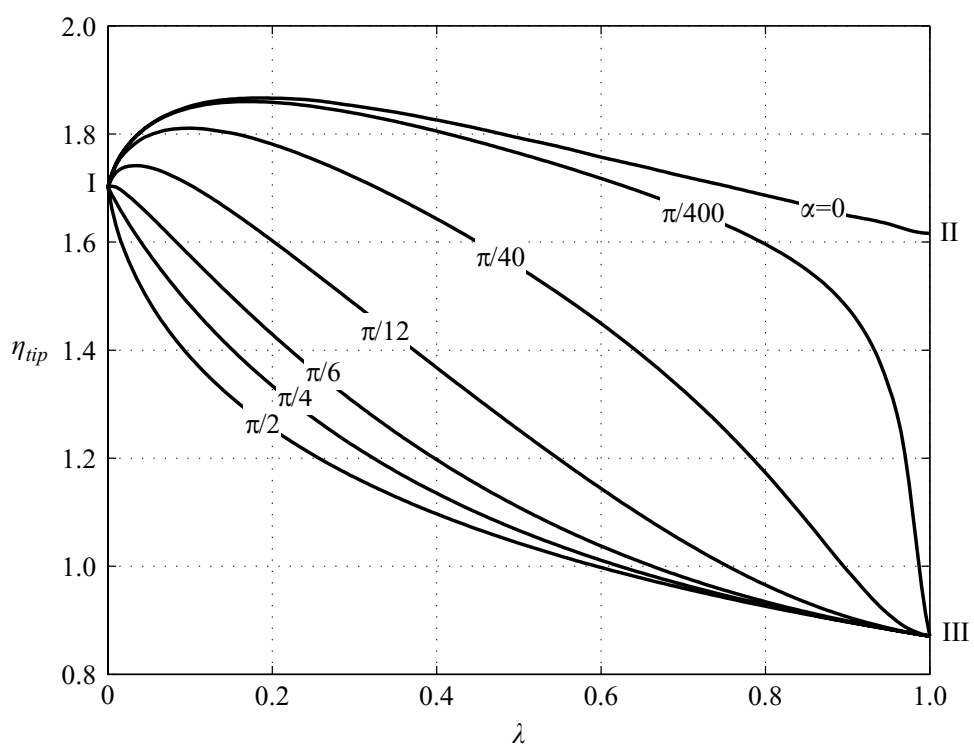

FIGURE 17. Meniscus-tip location $\eta_{t i p}(\lambda ; \alpha)$ : I, II, and III denote the results for the three asymptotic regimes.

and $(4.31 b)$ to

$$
[F(1-\lambda)+\lambda] F_{\eta \eta} \ll \frac{\sigma}{\mu} \frac{(1-\lambda)^{2}}{f^{2}} \frac{\langle\bar{w}\rangle^{*}}{H} \frac{T_{c}^{2}}{1+T_{c}^{2}} t .
$$

These relations provide a means of identifying in part the time domains for which the similarity solutions are valid. In general, both constraints will be satisfied over increasingly larger lengths of the liquid column as time increases. The low-inertia (3.3), low-gravity, and negligible-dynamic-contact-angle conditions may be quantified using the methods outlined in W\&L (see also Weislogel 1996).

Important quantities such as the dimensional tip length and the flow rate are gleaned from the numerical results. For example, $\eta_{t i p}$ and $F_{\eta}(0)$ are collected in figures 17 and 18 as functions of $\lambda$ for various $\alpha$. Typical data are also listed in tables 8 and 9. The results for the three asymptotic regimes are noted on the plots and are listed in table 7. The dimensional meniscus-tip location and volumetric flow rate may be written in closed form using these results:

$$
\begin{gathered}
\mathscr{L}=\eta_{t i p} G^{1 / 2} H^{1 / 2} t^{1 / 2}, \\
\dot{Q}=-F_{\eta}(0)\left(1-\lambda^{2}\right) F_{A} G^{1 / 2} H^{5 / 2} t^{-1 / 2},
\end{gathered}
$$

where

$$
G=\frac{\langle\bar{w}\rangle^{*} \sigma(1-\lambda)^{3} T_{c}^{2}}{\mu f\left(1+T_{c}^{2}\right)}
$$

and the descriptions of the relevant parameters can be found in table 1 . The forms of (4.33) and (4.34) allow rapid closed-form predictions of the tip location and flow rate for the purposes of system design and analysis (we also conjecture that such relations may be used for systems where $\theta_{c}=\theta \neq 0$ ).

Note that for certain values of $\alpha, \eta_{\text {tip }}$ is higher for non-zero $\lambda$. This does not mean that in dimensional form the meniscus tip moves faster in a rounded corner than in a sharp corner. In fact, it can be shown that $\mathscr{L}$ decreases monotonically with 


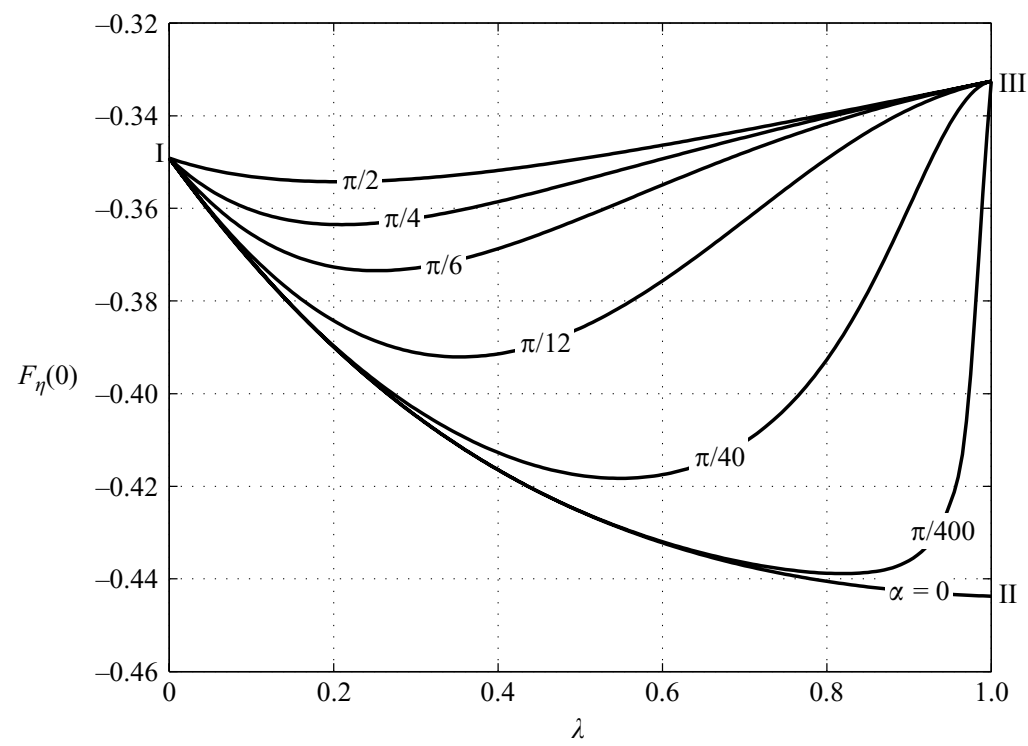

FigURE 18. $\left.F_{\eta}\right|_{\eta=0}(\lambda ; \alpha)$ : I, II, and III denote the results for the three asymptotic regimes.

\begin{tabular}{llllllll}
\hline$\lambda \backslash \alpha$ & 0.00000 & 0.00785 & 0.07854 & 0.26180 & 0.52360 & 0.78540 & 1.57080 \\
1.000 & 1.616125 & 0.870570 & 0.870570 & 0.870570 & 0.870570 & 0.870570 & 0.870570 \\
0.900 & 1.649297 & 1.478332 & 0.991580 & 0.906251 & 0.898595 & 0.897300 & 0.896724 \\
0.800 & 1.686416 & 1.596063 & 1.173220 & 0.965388 & 0.934378 & 0.928665 & 0.926051 \\
0.700 & 1.721637 & 1.664120 & 1.325698 & 1.046339 & 0.979965 & 0.966002 & 0.959334 \\
0.600 & 1.757264 & 1.717774 & 1.449343 & 1.143899 & 1.037531 & 1.011100 & 0.997672 \\
0.500 & 1.792822 & 1.764395 & 1.553318 & 1.252469 & 1.109254 & 1.066371 & 1.042685 \\
0.450 & 1.810237 & 1.785589 & 1.599801 & 1.309461 & 1.151082 & 1.098808 & 1.068434 \\
0.400 & 1.825860 & 1.805276 & 1.643109 & 1.367653 & 1.197224 & 1.135120 & 1.096915 \\
0.350 & 1.840322 & 1.823139 & 1.683309 & 1.426625 & 1.247918 & 1.175935 & 1.128728 \\
0.300 & 1.851980 & 1.838655 & 1.720193 & 1.485913 & 1.303392 & 1.222014 & 1.164703 \\
0.250 & 1.862371 & 1.850949 & 1.753175 & 1.544890 & 1.363834 & 1.274315 & 1.206029 \\
0.200 & 1.866357 & 1.858642 & 1.781100 & 1.602595 & 1.429360 & 1.334051 & 1.254517 \\
0.150 & 1.864622 & 1.859323 & 1.801767 & 1.657310 & 1.499869 & 1.402863 & 1.313153 \\
0.100 & 1.851401 & 1.848453 & 1.810772 & 1.705535 & 1.574650 & 1.483141 & 1.387538 \\
0.080 & 1.840725 & 1.838874 & 1.809146 & 1.721403 & 1.605269 & 1.519266 & 1.424116 \\
0.050 & 1.816387 & 1.815141 & 1.797339 & 1.738310 & 1.650722 & 1.578935 & 1.490975 \\
0.020 & 1.770559 & 1.770687 & 1.764311 & 1.738245 & 1.691633 & 1.647428 & 1.584800 \\
0.010 & 1.745089 & 1.745696 & 1.742862 & 1.729051 & 1.701580 & 1.673202 & 1.629717 \\
0.000 & 1.702110 & 1.702000 & 1.702000 & 1.702000 & 1.702000 & 1.702000 & 1.702000
\end{tabular}

TABLE 8. Tabulated values of $\eta_{\text {tip }}$.

increasing $\lambda$. However, $\mathscr{L}$ is sensitive to the choice of the constant $\langle\bar{w}\rangle^{*}$ for $\alpha \rightarrow 0$. The most intricate and accurate solution for $\mathscr{L}$ treats $\langle\bar{w}\rangle^{*}$ as a weakly varying function and solves (4.4) using curve-fit numerical data for $\langle\bar{w}\rangle^{*}$. Fortunately, it is found that evaluating $\langle\bar{w}\rangle^{*}$ at $h=0.5$ yields results that compare well (within $2 \%$ ) with second-order-polynomial curve-fit data. Select results for $\langle\bar{w}\rangle^{*}$ at $h=0.5$ are shown in figure 19 for this purpose. The evaluation of $\dot{Q}$ with $F_{\eta}(0)$ shown in figure 18 is 


\begin{tabular}{cccccccc}
\hline$\lambda \backslash \alpha$ & 0.00000 & 0.00785 & 0.07854 & 0.26180 & 0.52360 & 0.78540 & 1.57080 \\
1.000 & -0.443748 & -0.332584 & -0.332584 & -0.332584 & -0.332584 & -0.332584 & -0.332584 \\
0.900 & -0.442654 & -0.436063 & -0.359772 & -0.338644 & -0.336645 & -0.336305 & -0.336154 \\
0.800 & -0.440526 & -0.438781 & -0.392665 & -0.349311 & -0.341757 & -0.340335 & -0.339681 \\
0.700 & -0.437138 & -0.436485 & -0.410446 & -0.362616 & -0.347933 & -0.344683 & -0.343110 \\
0.600 & -0.432220 & -0.431970 & -0.417494 & -0.375665 & -0.354945 & -0.349305 & -0.346360 \\
0.500 & -0.425447 & -0.425379 & -0.417655 & -0.385869 & -0.362212 & -0.354049 & -0.349316 \\
0.450 & -0.421247 & -0.421231 & -0.415766 & -0.389311 & -0.365638 & -0.356367 & -0.350633 \\
0.400 & -0.416433 & -0.416454 & -0.412722 & -0.391420 & -0.368701 & -0.358561 & -0.351807 \\
0.350 & -0.410948 & -0.410993 & -0.408580 & -0.392067 & -0.371191 & -0.360530 & -0.352804 \\
0.300 & -0.404733 & -0.404792 & -0.403362 & -0.391146 & -0.372867 & -0.362137 & -0.353579 \\
0.250 & -0.397725 & -0.397791 & -0.397068 & -0.388566 & -0.373453 & -0.363201 & -0.354080 \\
0.200 & -0.389866 & -0.389930 & -0.389683 & -0.384254 & -0.372647 & -0.363482 & -0.354237 \\
0.150 & -0.381100 & -0.381157 & -0.381192 & -0.378151 & -0.370126 & -0.362667 & -0.353963 \\
0.100 & -0.371390 & -0.371433 & -0.371583 & -0.370232 & -0.365559 & -0.360358 & -0.353142 \\
0.080 & -0.367237 & -0.367273 & -0.367429 & -0.366558 & -0.363089 & -0.358908 & -0.352627 \\
0.050 & -0.360723 & -0.360746 & -0.360874 & -0.360520 & -0.358639 & -0.356056 & -0.351617 \\
0.020 & -0.353880 & -0.353890 & -0.353951 & -0.353880 & -0.353259 & -0.352269 & -0.350271 \\
0.010 & -0.351532 & -0.351536 & -0.351568 & -0.351544 & -0.351257 & -0.350774 & -0.349736 \\
0.000 & -0.349152 & -0.349152 & -0.349152 & -0.349152 & -0.349152 & -0.349152 & -0.349152
\end{tabular}

TABLE 9. Tabulated values of $\left.F_{\eta}\right|_{\eta=0}$.

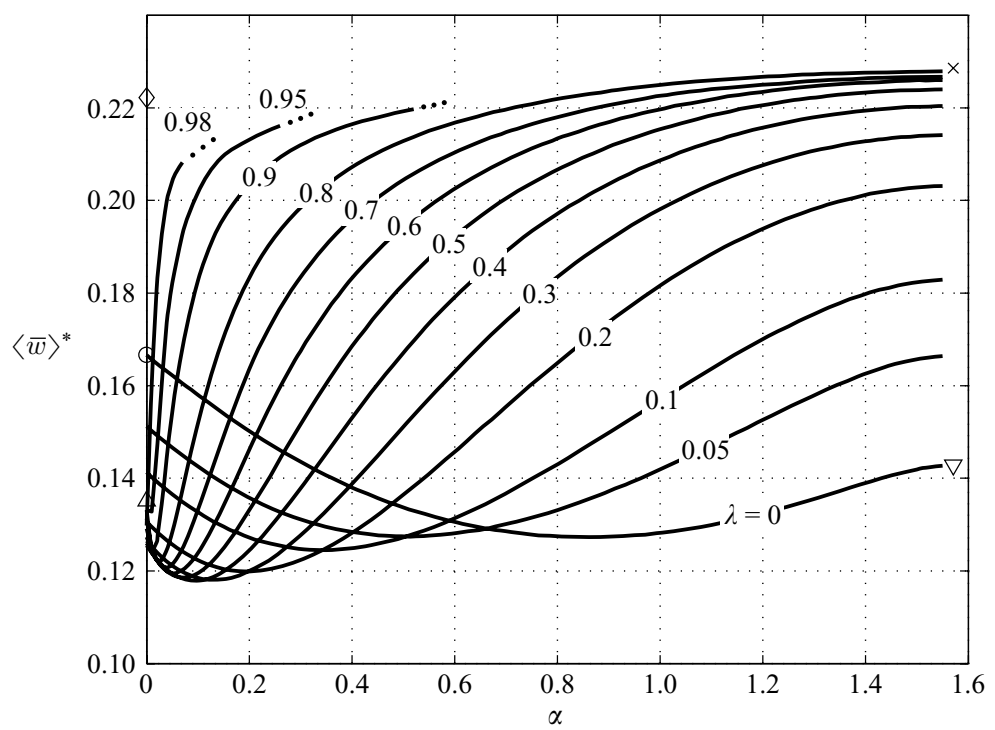

FIGURE 19. $\langle\bar{w}\rangle^{*}$ at $h=0.5$. The symbols represent asymptotic values also shown in figure 8 .

accurate to within $\pm 4 \%$ as compared with results obtained using a polynomial fit of $\langle\bar{w}\rangle^{*}$. In this way, $\langle\bar{w}\rangle^{*}$ may be effectively treated as a constant for a given geometry when it is evaluated at $h=0.5$. This method of estimating $\langle\bar{w}\rangle^{*}$ results in a significant simplification of the problem while incurring errors of less than $\pm 4 \%$.

Note that $H$ appears in both equations (4.33) and (4.34). This is the constant height $H$ for the flow according to W\&L. The method of de Lazzer et al. (1996) may be used to determine $H$ for right polygonal cylinders. A general approach was given by 
Finn \& Neel (1999). It is important to note that for a given polygonal cross-section, $H$ does not depend on the degree of corner roundedness $\lambda$.

\subsection{Imbibition in prewetted corners}

The imbibition problem addressed above may also be analyzed for flow advancing over a quiescent infinite column of the same fluid with uniform depth $H_{0}$, as studied numerically by Romero \& Yost (1996) for the case of flow in a sharp corner. Numerical and asymptotic analytic solutions are offered here for the rounded-corner problem. Such solutions are readily obtained numerically for the full similarity equation (4.25) with boundary conditions

$$
F(0)=1 \quad \text { and } \quad F(\infty)=1-\zeta,
$$

where $\zeta=1-H_{0} / H$. Typical solutions are those for the three asymptotic flow regimes given by $(4.29 a-c)$; some results are shown in figure 20 . The overall impact of corner roundedness is clear from inspection of the figure.

It is observed from $(4.29 a-c)$ that $F=$ constant is a non-trivial solution to the limiting governing equations, suggesting that asymptotic solutions for the three regimes are also possible. Introducing the asymptotic series

$$
F=F_{0}+\zeta F_{1}+\zeta^{2} F_{2}+O\left(\zeta^{3}\right)
$$

where $\zeta^{3} \ll 1$, and substituting (4.37) into $(4.29 a-c)$, yields respectively, $O(1), O(\zeta)$, and $O\left(\zeta^{2}\right)$ equations that are subject to boundary conditions as follows:

$$
\left.\begin{array}{rl}
O(1), & F_{0}=F_{0}(0)=F_{0}(\infty)=1 ; \\
O(\zeta), & F_{1}(0)=0, F_{1}(\infty)=-1 ; \\
O\left(\zeta^{2}\right), & F_{2}(0)=0, F_{2}(\infty)=0 .
\end{array}\right\}
$$

When solved, the equations produce asymptotic series for the three regimes accurate to $O\left(\zeta^{3}\right)$ :

I. $F=1-\zeta \operatorname{erf}\left(\frac{\eta}{\sqrt{2}}\right)$

$$
+\zeta^{2}\left\{\frac{1-e^{-\eta^{2}}}{\pi}+\frac{-\eta e^{-\eta^{2} / 2}+(1-1 / \pi) \sqrt{2 \pi}}{\sqrt{2 \pi}} \operatorname{erf}\left(\frac{\eta}{\sqrt{2}}\right)-\operatorname{erf}^{2}\left(\frac{\eta}{\sqrt{2}}\right)\right\},
$$

II. $F=1-\zeta \operatorname{erf}\left(\frac{\eta}{2}\right)$

$$
+\zeta^{2}\left\{\frac{1-e^{-\eta^{2} / 2}}{\pi}+\frac{\eta e^{-\eta^{2} / 4}+(\pi-2) / \sqrt{\pi}}{2 \sqrt{\pi}} \operatorname{erf}\left(\frac{\eta}{2}\right)-\frac{1}{2} \operatorname{erf}^{2}\left(\frac{\eta}{2}\right)\right\},
$$

III. $F=1-\zeta \operatorname{erf}\left(\frac{\eta}{2}\right)$

$$
+\zeta^{2}\left\{\frac{3\left(1-e^{-\eta^{2} / 2}\right)}{\pi}+\frac{-3 \eta e^{-\eta^{2} / 4}+(3 \pi-6) / \sqrt{\pi}}{2 \sqrt{\pi}} \operatorname{erf}\left(\frac{\eta}{2}\right)-\frac{3}{2} \operatorname{erf}^{2}\left(\frac{\eta}{2}\right)\right\} .
$$

Provided $\zeta^{3} \ll 1$, perhaps the most useful result of these solutions is the slope of the solution at $\eta=0$, from which the flow rate may be determined via (4.34). To $O\left(\zeta^{2}\right)$, 

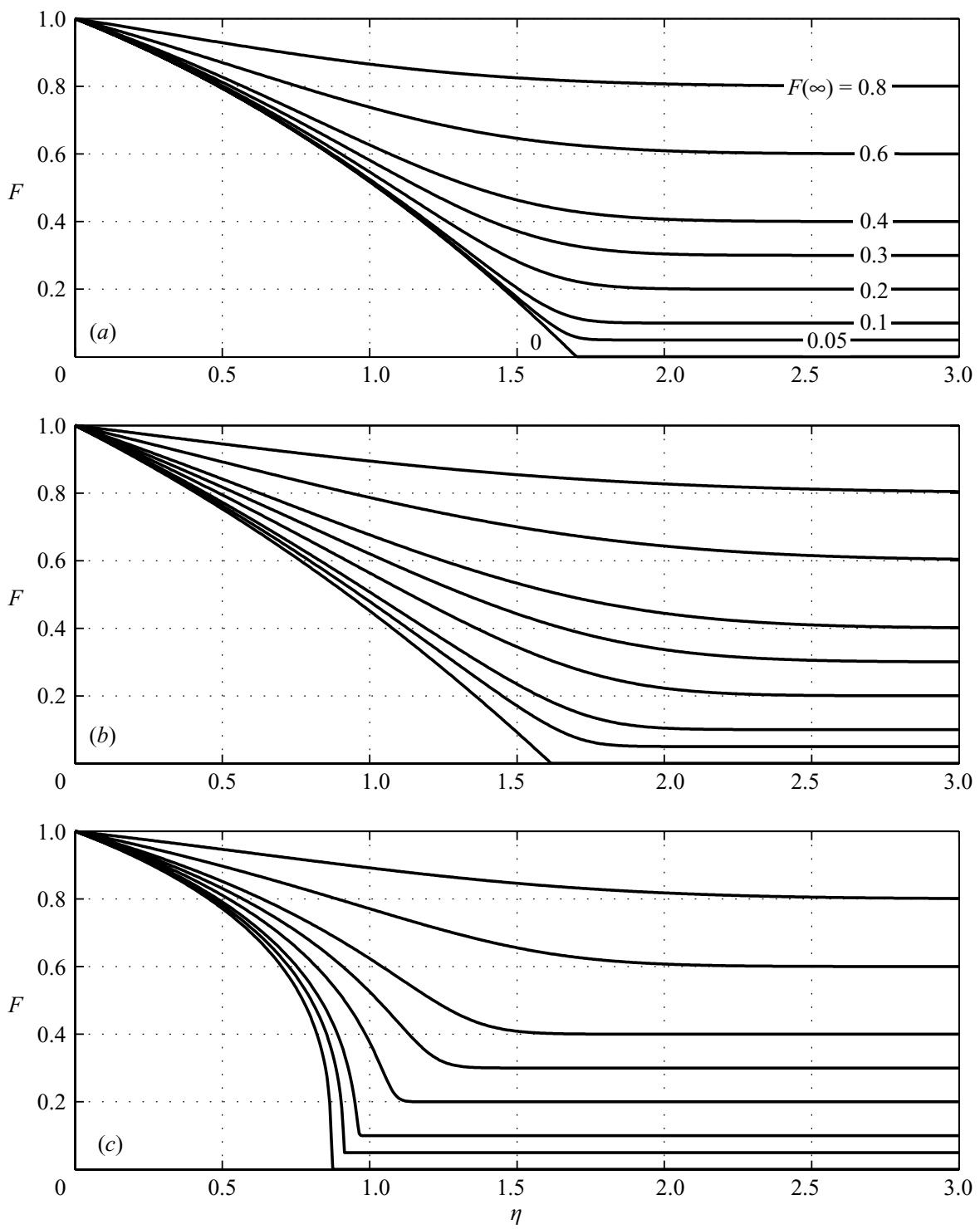

FIGURE 20. Finite-tip-curvature solutions in the three regimes: $(a)$ sharp-corner (I), (b) rectangular-section (II), and (c) thin-film (III).

(4.39) yields
I. Sharp corner
$F_{\eta}(0)=-0.798 \zeta+0.544 \zeta^{2}$,
II. Rectangular section $F_{\eta}(0)=-0.564 \zeta+0.103 \zeta^{2}$,
III. Thin film,
$F_{\eta}(0)=-0.564 \zeta+0.308 \zeta^{2}$,

which are presented in figure 21. The full numerical solutions fitted using second order polynomials are given for the three regimes. The percentage error of the asymptotic results (4.40) as compared with the numerical solutions of figure 21 are shown in figure 22. The asymptotic solutions for the sharp-corner and thin-film regimes are 


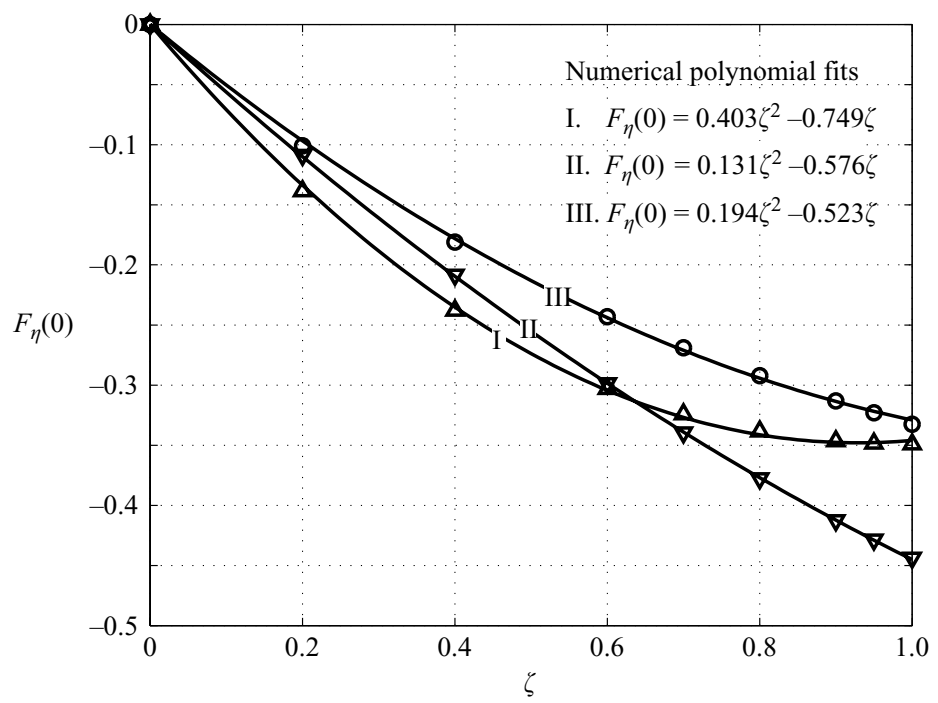

FIGURE 21. Numerical solutions of $F_{\eta}(0)$ for the three asymptotic flow regimes. The solid lines, (4.40), are second-order polynomial fits to the numerical results given in the figure.

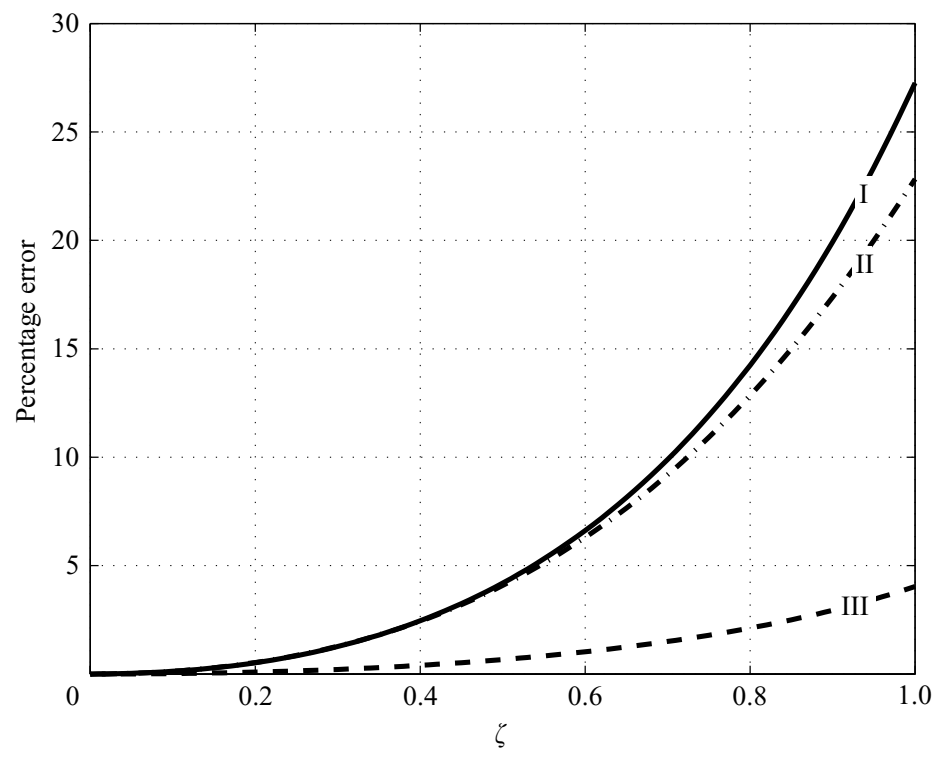

FIGURE 22. Percentage error between the asymptotic, (4.40), and numerical solutions of $F_{\eta}(0)$ for the three asymptotic flow regimes.

accurate to $\lesssim 10 \%$ for $\zeta$ up to 0.7 . It may be serendipitous that the discrepancies are $<5 \%$ for all $\zeta$ for the rectangular-section regime (III).

\section{Concluding remarks}

As an example of how analytical methods might be extended further to capillarydriven flow in rounded interior corners, several numerical and asymptotic analytic 
solutions have been presented in this paper:

(a) steady flows $(\S 4.3)$;

(b) perturbed infinite columns $(\$ 4.4)$;

(c) imbibition (initially dry corners) (§4.5);

(d) imbibition (initially wet corners) (\$4.6).

Closed-form expressions may be gleaned from such results, which are immediately useful to rapidly compute characteristics of the flow such as the flow rate, interface shape, and response time all as functions of the container geometry and fluid properties.

The solutions presented are formally restricted to perfectly wetting fluids in rounded corners that are tangent to planar walls. We conjecture that they may also be applied to rounded corners that intersect planar walls at the contact angle $\left(\theta_{c}=\theta>0\right)$ provided that the Concus-Finn critical corner-wetting condition is satisfied. General solutions in terms of specific corner rounding and arbitrary contact angle require an alternate scaling method not addressed in this work.

The analytical results are enabled by a specific geometric scaling of the cross-flow problem that renders the subsequent governing equation only weakly dependent on the numerical data. A significant portion of this paper has been devoted to both local and global scale analysis $(\S \S 3.1 .1-3.1 .3)$. For all values of corner roundedness $0 \leqslant \lambda \leqslant 1$, fluid depth $0 \leqslant h^{*} \leqslant 1$, and corner half-angle $0 \leqslant \alpha \leqslant \pi / 2$, with $\theta=0$, the numerical coefficient is banded such that $0.117 \leqslant\langle\bar{w}\rangle^{*} \leqslant 0.229$. As suggested herein, by applying the value of $\langle\bar{w}\rangle^{*}$ at $h^{*}=0.5$ (from figure 19), $\langle\bar{w}\rangle^{*}$ may be treated effectively as a constant with errors expected to be $\lesssim \pm 4 \%$ and in most cases $\lesssim \pm 2 \%$. The wide majority of the geometric dependence of the viscous resistance of the flow is thus captured by the scaling. Such solutions are ideally suited for geometric optimizations of the flow. They are also compact and may often be interrogated by inspection.

Several salient features of the solutions are collected in summary.

(i) An intricate nonlinear evolution equation for the interface height $h$ was derived (4.22) for rounded-corner flows. From this equation, three nonoverlapping regimes are readily identified: (I) the sharp-corner regime with $\bar{\lambda} \ll 1$, (II) the narrow-corner rectangular-section regime with $\bar{\lambda} \gg 1$ and $\bar{\lambda}^{2} \alpha^{2} \ll 1$, and (III) the thin-film regime with $\bar{\lambda} \gg 1$ and $\bar{\lambda}^{2} \tan ^{2} \alpha \gg 1$.

(ii) Numerical steady solutions were obtained in general with analytic solutions for the three regimes.

(iii) As corner roundedness increases, the response time of a perturbed column of fluid in a rounded corner decreases with decreasing corner half-angle $\alpha$.

(iv) The presence of finite corner roundedness for flows with advancing fronts leads to an infinite slope at the leading edge. In this (thin-film) region the lubrication and negligible-streamwise-curvature assumptions are violated. However, these constraints were shown to be increasingly valid as time progresses.

(v) A numerical similarity solution was solved for the 23-term nonlinear evolution equation for the imbibition problem. Results for both initially dry and prewetted sharp and rounded corners were presented with important coefficients determined for closed-form equations predicting important features of the flow. Asymptotic solutions for the prewet problem were solved to second order; these are helpful in estimating the flow rate and speed of the advancing front.

This work was supported by the NASA Office of Biological and Physical Research through contract NNC05AA29A monitored by E. Ramé and A. Wilkinson. As 
usual, we wish to thank P. Concus for helpful discussions concerning critical wetting conditions.

\section{Appendix. Asymptotic-solution details for $\langle\bar{w}\rangle^{*}$}

Case I. The narrow-corner limit, ${\overline{T_{c}}}^{2} \ll 1$

Because $\bar{T}_{c}$ consists of two terms, the narrow-corner limit ${\overline{T_{c}}}^{2} \ll 1$ requires that both conditions $\alpha^{2} \ll 1$ and $\bar{\lambda} \lesssim 1$ are met. The latter condition is satisfied for intermediate to low values of $\lambda \neq 1$ and $O(1)$ values of $h^{*}$ but cannot be satisfied for small but finite $\alpha$ with either $h^{*} \rightarrow 0$ or $\lambda \rightarrow 1$, since from (3.13) $\bar{\lambda} \equiv \lambda / h^{*}(1-\lambda)$.

For the case ${\overline{T_{c}}}^{2} \ll 1$, the dimensionless local velocity $\bar{w}^{*}$ can be represented by the expansion

$$
\bar{w}^{*}=\bar{w}_{0}^{*}+\bar{T}_{c}^{2} \bar{w}_{1}^{*}+O\left(\bar{T}_{c}^{4}\right) .
$$

The quantities $\bar{P}^{*}$ and $\bar{S}^{*}$ may be expanded similarly. Equation (A 1) when substituted into (3.14) yields the zeroth-order equation

$$
1=\frac{\partial^{2} \bar{w}_{0}^{*}}{\partial \bar{y}^{* 2}}
$$

subject to the boundary conditions (3.15)-(3.18), the last of which does not apply at leading order.

Note. In the special case of a predominantly flat fluid interface where $\partial \bar{S}^{*} / \partial \bar{y}^{*} \sim \bar{T}_{c}^{4}$, (3.18) reduces to the $O\left(\bar{T}_{c}^{2}\right)$ equation $\partial \bar{w}_{o}^{*} / \partial \bar{x}^{*}=0$. In this situation all the boundary conditions (3.15)-(3.18) are satisfied to $O\left(T_{c}^{2}\right)$. Note also that $\bar{S}^{*}=\bar{S}_{0}^{*}+O\left(\bar{T}_{c}^{4}\right)$, as shown in figure 6 .

Solving (A 2) produces

$$
\left.\begin{array}{ll}
\bar{w}_{0_{1}}^{*}=\frac{1}{2}\left[\bar{y}^{* 2}-\left(\bar{S}_{\mathrm{w}_{1}}^{*-1}\left(\bar{x}^{*}\right)\right)^{2}\right] & \text { on } 0 \leqslant \bar{x}^{*} \leqslant \bar{x}_{p}^{*} \\
\bar{w}_{0_{2}}^{*}=\frac{1}{2}\left[\bar{y}^{* 2}-\left(\bar{S}_{\mathrm{w}_{2}}^{*-1}\left(\bar{x}^{*}\right)\right)^{2}\right] & \text { on } \quad \bar{x}_{p}^{*}<\bar{x}^{*} \leqslant \bar{x}_{m}^{*} .
\end{array}\right\}
$$

The local average velocity $\left\langle\bar{w}_{0}\right\rangle_{I}^{*}$ may in turn be evaluated via

$$
\left\langle\bar{w}_{0}\right\rangle_{I}^{*}=\frac{1}{\bar{A}^{*}}\left(\int_{0}^{\bar{x}_{p}^{*}} \int_{0}^{{\overline{w_{w}}}_{1}^{* 1}} \bar{w}_{0_{1}}^{*} \mathrm{~d} \bar{y}^{*} \mathrm{~d} \bar{x}^{*}+\int_{\bar{x}_{p}^{*}}^{1} \int_{0}^{\bar{s}_{\mathrm{w}_{2}}^{*-1}} \bar{w}_{0_{2}}^{*} \mathrm{~d} \bar{y}^{*} \mathrm{~d} \bar{x}^{*}+\int_{1}^{\bar{x}_{m}^{*}} \int_{\bar{S}_{0}^{*-1}}^{S_{w_{2}}^{*-1}} \bar{w}_{0_{2}}^{*} \mathrm{~d} \bar{y}^{*} \mathrm{~d} \bar{x}^{*}\right) .
$$

For narrow corners with $\alpha^{2} \ll 1$ and with relatively small roundedness $\bar{\lambda} \ll 1,\left\langle\bar{w}_{0}\right\rangle_{I}^{*}$ reduces to

$$
\left\langle\bar{w}_{0}\right\rangle_{I a}^{*}=\frac{1}{6}+\left(\frac{16-5 \pi}{48}\right) \alpha+\left(\frac{14-\pi}{6}\right) \bar{\lambda}+O\left(\alpha^{2}, \bar{\lambda}^{2}\right),
$$

which recovers the zeroth-order solution $\langle\bar{w}\rangle^{*}=F_{I a}=1 / 6$ of W\&L for the sharp corner defined by $\alpha=0$ and $\lambda=0$. This narrow-sharp-corner domain is shown in figure $7(a)$.

A second narrow-corner limit is possible for the case $\alpha^{2} \bar{\lambda}^{2} \ll 1$, with $\bar{\lambda}^{2} \gg 1$. Defining $\chi \equiv 1 / \bar{\lambda},\left\langle\bar{w}_{0}\right\rangle_{I}^{*}$ reduces to

$$
\begin{array}{r}
\left\langle\bar{w}_{0}\right\rangle_{I b}^{*}=\frac{4}{3 \pi^{2}}+\left(\frac{256+216 \pi-37 \pi^{2}}{48 \pi^{3}}\right) \alpha+\left(\frac{-16+16 \pi}{3 \pi^{3}}\right) \chi-\frac{\alpha}{8 \pi \chi} \\
+\frac{104-3 \pi^{2}}{48 \pi^{2}} \frac{\alpha^{2}}{\chi}+O\left(\alpha^{2}, \alpha \chi, \chi^{2}, \alpha^{3} / \chi\right),
\end{array}
$$


which is valid for $\chi^{2} \ll 1$. This narrow-corner-rectangular-section domain is shown in figure $7(b)$. At zeroth order, $\left\langle\bar{w}_{0}\right\rangle_{I b}^{*}=4 / 3 \pi^{2}$.

Case II. The thin-film limit, ${\overline{T_{c}}}^{2} \gg 1$

In a similar manner to that above, $\langle\bar{w}\rangle^{*}$ may be determined for thin-film flows where $\bar{T}_{c}^{2} \gg 1$. From two terms defining $\bar{T}_{c}$, this situation arises when either $\tan ^{2} \alpha \gg 1$ (i.e. $\alpha \rightarrow \pi / 2$ ) or $\bar{\lambda}^{2} \tan ^{2} \alpha \gg 1$ (i.e. $\bar{\lambda} \rightarrow \infty$ ) or when both these conditions hold. Here there are three limiting values for $\langle\bar{w}\rangle^{*}$ that may be determined. By expanding $\bar{w}^{*}$,

$$
\bar{w}^{*}=\bar{w}_{0}^{*}+\frac{1}{{\overline{T_{c}}}^{2}} \bar{w}_{1}^{*}+O\left(\frac{1}{{\overline{T_{c}}}^{4}}\right),
$$

as well as $\bar{P}^{*}$ and $\bar{S}^{*}$, the zeroth-order equation (3.14) reduces to

$$
1=\frac{\partial^{2} \bar{w}_{0}^{*}}{\partial \bar{x}^{* 2}}
$$

The no-slip boundary condition is given by (3.15), and the free-surface shear-stress condition given by $(3.18)$ reduces to

$$
\frac{\partial \bar{w}_{0}^{*}}{\partial \bar{x}^{*}}=0 \quad \text { on } \bar{x}^{*}=\bar{S}_{0}^{*} .
$$

Again, the solution domain is divided into two regions $0 \leqslant \bar{y}^{*} \leqslant \bar{y}_{p}^{*}$ and $\bar{y}_{p}^{*}<\bar{y}^{*} \leqslant \bar{y}_{m}^{*}$ over which (A 8 ) is solved. Introducing $\Omega \equiv \pi / 2-\alpha$, under the conditions $\Omega^{2} \ll 1$ and $\bar{\lambda}^{2} \ll 1$, it is found that

$$
\left\langle\bar{w}_{0}\right\rangle_{\text {II } a}^{*}=\frac{1}{7}+\frac{2}{7} \bar{\lambda}+O\left(\Omega^{2}, \bar{\lambda}^{2}\right) .
$$

When $\lambda=0$ the wide-angle sharp-corner result of W\&L is recovered, where at zeroth order $\left\langle\bar{w}_{0}\right\rangle_{I I a}^{*}=F_{I I}=1 / 7$. As for case I, $\left\langle\bar{w}_{0}\right\rangle_{I I a}^{*}$ is independent of $h^{*}$ to leading order provided $\bar{\lambda} \ll 1$. This thin film-condition with wide corner angle and slight corner roundedness is shown in figure $7(c)$.

Under the conditions $\Omega^{2} \ll 1$ and $\bar{\lambda}^{2} \gg 1$,

$$
\left\langle\bar{w}_{0}\right\rangle_{I I b}^{*}=\frac{8}{35}+O\left(\Omega^{2}, \chi^{2}\right) \text {. }
$$

This case corresponds to the wide-corner-angle and large-roundedness condition, which is shown in figure $7(d)$. This expression for $\left\langle\bar{w}_{0}\right\rangle_{I I b}^{*}$ is valid for all $0 \leqslant h^{*} \leqslant 1$ and at zeroth order $\langle\bar{w}\rangle_{I I b}^{*}=8 / 35$.

It is also possible to satisfy the condition ${\overline{T_{c}}}^{2} \gg 1$ for any value of $\alpha$ when $\bar{\lambda}^{2} \tan ^{2} \alpha \gg 1$. In such cases, (A 8) applies but to the radial problem shown in figure 7(e), which is 'unwrapped' and solved in a Cartesian domain. To leading order the profile of the free surface is

$$
x=\frac{(\cos (y / R)-\sin \alpha) h}{1-\sin \alpha}, \quad y \in[R(\alpha-\pi / 2), R(\pi / 2-\alpha)] .
$$

Solving (A 8) over the domain gives rise to

$$
\left\langle\bar{w}_{0}\right\rangle^{*}=F_{w}(\alpha)+F_{w_{1}}(\alpha) \chi+\ldots,
$$

with

$$
F_{w}(\alpha)=\frac{-11 \cos 3 \alpha+27 \cos \alpha+3(\pi-2 \alpha)(\sin 3 \alpha-9 \sin \alpha)}{72(1-\sin \alpha)^{2}(\cos \alpha+(\alpha-\pi / 2) \sin \alpha)},
$$


which is a very weak function of $\alpha$, validating the unwrapped Cartesian solution. From (A 11) and (A 14) it is seen that

$$
F_{w}(0)=\frac{2}{9} \leqslant F_{w}(\alpha) \leqslant \frac{8}{35}=F_{w}\left(\frac{\pi}{2}\right) .
$$

For the narrow-corner thin-film case, with $\alpha^{2} \ll 1$ and $\bar{\lambda}^{2} \tan ^{2} \alpha \gg 1$, (A 13) reduces to

$$
\begin{aligned}
& \left\langle\bar{w}_{0}\right\rangle_{I I c}^{*}=\frac{2}{9}+\frac{128-4 \pi-11 \pi^{2}}{576} \chi+\frac{16-5 \pi}{36} \alpha-\frac{\pi}{288} \frac{\chi}{\alpha} \\
+ & \frac{20480-3200 \pi^{2}+576 \pi^{3}+44 \pi^{4}-5 \pi^{5}-15 \pi^{6}}{5760 \pi^{3}} \frac{\chi^{2}}{\alpha}+O\left(\alpha^{2}, \chi^{2} / \alpha^{2}, \chi \alpha\right)
\end{aligned}
$$

with zeroth-order solution $\langle\bar{w}\rangle_{I I c}^{*}=2 / 9$. All the asymptotic solutions for $\langle\bar{w}\rangle^{*}$ are plotted in figure 8 .

Case III. Hydraulic diameter

For completeness, applying the hydraulic-diameter method to this problem gives rise to

$$
\left\langle\bar{w}_{0}\right\rangle_{h y d}^{*}=\frac{1}{8} \frac{1+\bar{T}_{c}^{2}}{\bar{T}_{c}^{2}}\left[\frac{F_{A}}{f}\left(\frac{1+2 \bar{\lambda}}{\cot \alpha+\delta \bar{\lambda}}\right)\right]^{2}
$$

with zeroth-order solutions $\left\langle\bar{w}_{0}\right\rangle_{h y d}^{*}=1 / 8,2 / \pi^{2}, 1 / 18,2 / 9,2 / \pi^{2}$ for conditions Ia, Ib, IIa, IIb, and IIc respectively, as listed in table 4 . At a minimum, $1 / 18 \lesssim\left\langle\bar{w}_{0}\right\rangle_{h y d}^{*} \lesssim 2 / 9$.

\section{REFERENCES}

Al-Futaisi, A. \& PatzeK, T. W. 2002 Three-phase hydraulic conductances in angular capillaries. In SPE 75193, presented at the SPE/DOE 13th Symp. on Improved Oil Recovery, Tulsa OK, April 2002.

Ayyaswamy, P. S., Catton, I. \& Edwards, D. K. 1974 Capillary flow in triangular grooves. Trans ASME: J. Appl. Mech. 41, 332-336.

BICO, J. \& QUÉRÉ, D. 2002 Rise of liquids and bubbles in angular capillary tubes. J. Colloid Interface Sci. 247, 162-166.

Concus, P. \& Finn, R. 1969 On the behavior of capillary surface in a wedge. Proc. Natl. Acad. Sci. USA 63 (2), 292-299.

Concus, P. \& FinN, R. 1990 Dichotomous behavior of capillary surfaces in zero gravity. Microgravity Sci. Technol. III (2), 87-92.

Concus, P., Finn, R. \& Weislogel, M. M. 1996 Proboscis container shapes for the USML-2 interface configuration experiment. In Materials and Fluids under Low Gravity (eds. L. Ratke, H. Walter \& B. Feuerbacher). Lecture Notes in Physics, vol. 464, pp. 261-270. Springer.

Cox, S. J. \& Verbist, G. 2003 Liquid flow in foams under microgravity. Microgravity Sci. Technol. XIV, 45-52.

Cubard, T. \& Ho, C.-M. 2004 Transport of bubbles in square microchannels. Phys. Fluids 16 (12), 4575-4585.

Darhuber, A. A. \& Troian, S. M. 2005 Principles of microfluidic actuation by modulation of surface stresses. Ann. Rev. Fluid Mech. 37, 425-455.

Darhuber, A. A., Troian, S. M. \& Reisner, W. W. 2001 Dynamics of capillary spreading along hydrophilic microstripes. Phys. Rev. E 64 (3), 031603:1-8.

de Lazzer, A., Langbein, D., Dreyer, M. \& Rath, H.-J. 1996 Mean curvature of liquid surfaces in cylindrical containers of arbitrary cross-section. Microgravity Sci. Technol. IX (3), 208-219.

Dong, M. \& Chatzis, I. 1995 The imbibition and flow of a wetting liquid along the corners of a square capillary tube. J. Colloid Interface Sci. 172, 278-288.

Finn, R. \& NeEL, R. W. 1999 C-singular solutions of the capillary problem. Z. Reine Ange. Math. 512, $1-25$. 
FusITA, F. 1952 The exact pattern of a concentration-dependent diffusion in a semi-infinite medium, parts i and ii. Textile Res. J. 22, 757-760, 823-827.

Kim, E. \& Whitesides, G. M. 1997 Imbibition and flow of wetting liquids in noncircular capillaries. J. Phys. Chem. B 101, 855-863.

Kistler, S. F. 1993 Hydrodynamics of wetting. In Wettability, chap. 6 pp. 311-429. Marcel Dekker.

LENORMAND, R. \& ZARCONE, C. 1984 Roles of roughness and edges during imbibition in square capillaries. In SPE 13264, presented at the 59th Annual Meeting of the SPE. Houston TX, September 1984.

Mann, J. A. J., Romero, L., Rye, R. R. \& Yost, F. G. 1995 Flow of simple liquids down narrow $\bigvee$ grooves. Phys. Rev. E 52 (4), 3967-3972.

Mayer, F. J., McGrath, J. F. \& Steele, J. W. 1983 A class of similarity solutions for the nonlinear thermal conduction problem. J. Phys. A: Math. Gen. 16, 3393-3400.

Nehari, Z. 1975 Conformal Mapping. Dover.

Peterson, G. P. \& HA, J. M. 1998 Capillary performance of evaporating flow in micro grooves: an approximate analytical approach and experimental investigation. Trans. ASME J: J. Heat Transfer 120, 743-751.

Ransohoff, T. C., Gauglitz, P. A. \& Radke, C. J. 1987 Snap-off of gas bubbles in smoothly constricted noncircular capillaries. AICHE J. 33 (5), 753-765.

Ransohoff, T. C. \& RADKe, C. J. 1988 Laminar flow of a wetting liquid along the corners of a predominantly gas-occupied noncircular pore. J. Colloid Interface Sci. 121, 392-401.

Romero, L. A. \& Yost, F. G. 1996 Flow in an open channel capillary. J. Fluid Mech. 322, 109-129.

TChiKanda, S. W., Nilson, R. H. \& Griffiths, S. K. 2004 Modeling of pressure and shear-driven flows in open rectangular microchannels. Int. J. Heat Mass Transfer 47, 527-538.

Tuller, M. \& OR, D. 2001 Hydraulic conductivity of variably saturated porous media: film and corner flow in angular pore space. Water Resour. Res. 37 (5), 1257-1276.

Verbist, G., Weaire, D. \& Kraynik, A. M. 1996 The foam drainage equation. J. Phys.: Condens. Matter 8, 3715-3731.

Weislogel, M. M. 1996 Capillary flow in an interior corner. PhD thesis, Northwestern University.

Weislogel, M. M. 2001 a Capillary flow in containers of polygonal section. AIAA J. 39 (12), $2320-2326$.

Weislogel, M. M. $2001 \mathrm{~b}$ Capillary flow in interior corners: the infinite column. Phys. Fluids 13 (11), 3101-3107.

WeISLOGEL, M. M. 2003 Some analytical tools for fluids management in space: isothermal capillary flows along interior corners. Adv. Space Res. 32 (2), 163-170.

Weislogel, M. M. \& Collicott, S. H. 2004 Capillary rewetting of vaned containers: spacecraft tank rewetting following thrust resettling. AIAA J. 42 (12), 2551-2561.

Weislogel, M. M. \& Lichter, S. 1996 A spreading drop in an interior corner: theory and experiment. Microgravity Sci. Technol. IX (3), 175-184.

Weislogel, M. M. \& Lichter, S. 1998 Capillary flow in an interior corner. J. Fluid Mech. 373, 349-378.

Zhou, D., Blunt, M. \& OrR, F. M. J. 1997 Hydrocarbon drainage along corners of noncircular capillaries. J. Colloid Interface Sci. 187, 11-21. 